\title{
Application of Gradient Boosting Machine Learning Algorithms to Predict Uniaxial Compressive Strength of Soft Sedimentary Rocks at Thar Coalfield
}

\author{
Niaz Muhammad Shahani ${ }^{\mathbb{D}},{ }^{1,2}$ Muhammad Kamran ${ }^{\mathbb{D}},{ }^{3}$ Xigui Zheng $\left(\mathbb{D},{ }^{1,2,4,5}\right.$ \\ Cancan Liu $(\mathbb{D}),{ }^{1,2}$ and Xiaowei Guo $\mathbb{D D}^{1,2}$ \\ ${ }^{1}$ School of Mines, China University of Mining and Technology, Xuzhou 221116, Jiangsu Province, China \\ ${ }^{2}$ Key Laboratory of Deep Coal Resources Mining, Ministry of Education of China, School of Mines, \\ China University of Mining \& Technology, Xuzhou 221116, China \\ ${ }^{3}$ Bandung Institute of Technology, Indonesia \\ ${ }^{4}$ School of Mines and Civil Engineering, Liupanshui Normal University, Liupanshui 553004, China \\ ${ }^{5}$ Guizhou Guineng Investment Co., Ltd., Liupanshui 553600, China \\ Correspondence should be addressed to Xigui Zheng; fs16020006@cumt.edu.cn
}

Received 18 August 2021; Revised 6 October 2021; Accepted 22 October 2021; Published 1 November 2021

Academic Editor: Zhoujing Ye

Copyright (c) 2021 Niaz Muhammad Shahani et al. This is an open access article distributed under the Creative Commons Attribution License, which permits unrestricted use, distribution, and reproduction in any medium, provided the original work is properly cited.

\begin{abstract}
The uniaxial compressive strength (UCS) of rock is one of the essential data in engineering planning and design. Correctly testing UCS of rock to ensure its accuracy and authenticity is a prerequisite for assuring the design of any rock engineering project. UCS of rock has a broad range of applications in mining, geotechnical, petroleum, geomechanics, and other fields of engineering. The application of the gradient boosting machine learning algorithms has been rarely used, especially for UCS prediction, and has performed well, based on the relevant literature of the study. In this study, four gradient boosting machine learning algorithms, namely, gradient boosted regression (GBR), Catboost, light gradient boosting machine (LightGBM), and extreme gradient boosting (XGBoost), were developed to predict the UCS in MPa of soft sedimentary rocks of the Block-IX at Thar Coalfield, Pakistan, using four input variables such as wet density $\left(\rho_{\mathrm{w}}\right)$ in $\mathrm{g} / \mathrm{cm}^{3} ;$ moisture in $\%$; dry density $\left(\rho_{\mathrm{d}}\right)$ in $\mathrm{g} / \mathrm{cm}^{3}$; and Brazilian tensile strength (BTS) in MPa. Then, 106-point dataset was allocated identically for each algorithm into $70 \%$ for the training phase and $30 \%$ for the testing phase. According to the results, the XGBoost algorithm outperformed the GBR, Catboost, and LightGBM with coefficient of correlation $\left(R^{2}\right)=0.99$, mean absolute error (MAE) $=0.00062$, mean square error $(\mathrm{MSE})=0.0000006$, and root mean square error $(\mathrm{RMSE})=0.00079$ in the training phase and $R^{2}=0.99, \mathrm{MAE}=0.00054, \mathrm{MSE}=0.0000005$, and $\mathrm{RMSE}=0.00069$ in the testing phase. The sensitivity analysis showed that BTS and $\rho_{\mathrm{w}}$ are positively correlated, and the moisture and $\rho_{\mathrm{d}}$ are negatively correlated with the UCS. Therefore, in this study, the XGBoost algorithm was shown to be the most accurate algorithm among all the investigated four algorithms for UCS prediction of soft sedimentary rocks of the Block-IX at Thar Coalfield, Pakistan.
\end{abstract}

\section{Introduction}

The uniaxial compressive strength (UCS) of rock is one of the important data in engineering planning and design. Correctly testing UCS of rock to ensure its accuracy and authenticity is a prerequisite for ensuring the design of any rock engineering project. UCS of rock has a wide range of applications in mining, geotechnical, petroleum, geomechanics, and other fields of engineering. The study of rock mechanical properties is the basis for innovative advances associated with energy supply. The significance of rock mechanics is acknowledged in the advancement of natural assets, for example, the protection of energy sources (petroleum products: oil, coal, and natural gas), as well as the 
protection of the surrounding rock environment. In addition, protective waste removal and hydroelectric energy assets require further examination of rocks and soils, which are dependent on the mechanical properties of rocks $[1,2]$. Unconfined UCS of rocks plays a significant role in tackling issues associated with rock mechanics and the design of geostructures [3-6]. Various researchers have proposed empirical equations in order to estimate the UCS of different rock mass databases as tabulated in Table 1.

Some researchers have employed indirect testing methods, i.e., multiple linear regression (MLR) [17, 22, 23], artificial neural network (ANN) [23, 24], adaptive neurofuzzy inference system (ANFIS) [25], and other machine learning algorithms to estimate the accuracy and reliability of rock data [26-28], rather than using direct tests recommended by international standards, which are considered time-consuming, expensive, and unreliable [29, 30]. While these frameworks are appropriate, fast, and favorable techniques to tackle difficult problems, in most cases, they are simply capable of understanding the complex interactions among variables to estimate an objective and do not provide any intuition about the interrelationships among predictors and a return value [31]. Abidi et al. predicted the UCS of intact rocks with 196 data points, i.e., sandstone, marl, limestone, and conglomerate, using the intelligent ANN method and MLR. According to the results of their study, the ANN model performed better than the MLR model in terms of correlation coefficient $\left(R^{2}\right)$, mean absolute error (MAE), and root mean square error (RMSE) [23]. Abidi et al. employed both ANN and ANFIS models to predict UCS of 136 sandstone rock samples because laboratory testing methods are time-consuming and complex. Based on the results of several performance metrics, i.e., $R^{2}$, RMSE, and variance accounted for (VAF), the ANFIS model showed the best performance over ANN [32]. Ceryan et al. adopted soft computing methods, i.e., extreme learning machine (ELM) and minimax probability machine regression (MPMR), to predict the UCS of volcanic rocks. In addition, the least-square support vector machine (LS-SVM) model was incorporated to compare its performance with the proposed soft computing models. Thus, ELM and MPMR outperformed the LS-SVM [33]. Ceryan et al. developed the fuzzy interface system (FIS), ANN, and LV-SVM to predict the UCS of rocks. According to their results, LVSVM performed better than the other developed models [34]. Neurogenetic and multiple regression (MR) methods were developed by Monjezi et al. to estimate the UCS of rocks. The neurogenetic approach was best applied compared to MR [35]. Aboutaleb et al. predicted the UCS of carbonate rocks using nondestructive tests, namely, simple regression analysis (SRA), multiple regression analysis (MRA), ANN, and support vector regression (SVR). As reported by the SVR model, it performed best among all the models studied [36]. Ghasemi et al. developed a soft computing (model tree) approach to predict the UCS of carbonate rocks, which proved to have the best performance [37]. Barzegar et al. used ensemble tree-based machine learning approaches such as "random forest (RF), M5 model tree, and multivariate adaptive regression splines (Mars)" for predicting UCS of travertine rocks. In parallel, an ensemble committee-based ANN model was developed to correlate the returns of proposed models to determine the predicted UCS in accuracy. According to the results, Mars outperformed all studied models [38]. Matin et al. used RF for variable selection and UCS prediction, and the proposed RF for UCS prediction was satisfactory [39]. Zhong et al. predicted rock mechanical property, i.e., UCS with an established XGBoost model of machine learning, which proved to have the best performance in results [40].

According to the above literature, the application of the gradient boosting machine learning algorithms has been rarely used for UCS prediction. In this work, we proposed an innovative adaptation of four gradient boosting machine learning algorithms such as gradient boosted regression (GBR), Catboost, light gradient boosting machine (LightGBM), and extreme gradient boosting (XGBoost) that improves the handling of concept drift. The database employed in this work has been collected from soft sedimentary rocks of the Block-IX at Thar Coalfield, Pakistan. Previously, researchers considered wet density $\left(\rho_{\mathrm{w}}\right)$ [41]; moisture [41]; dry density $\left(\rho_{\mathrm{d}}\right)$ [42]; and Brazilian tensile strength (BTS) [43] as input variables for evaluating the UCS. Based on the literature, the four significant input parameters $\rho_{\mathrm{w}}$ in $\mathrm{g} / \mathrm{cm}^{3}$; moisture in $\%$; dry $\rho_{\mathrm{d}}$ in $\mathrm{g} / \mathrm{cm}^{3}$; and BTS in $\mathrm{MPa}$ are used for each developed algorithm. Moreover, 106-point dataset was allocated identically for each algorithm into $70 \%$ for the training phase and $30 \%$ for the testing phase. Figure 1 illustrates the flowchart of this study.

\section{Overview of the Study Area and Dataset}

Thar Coalfield is the 7th world's biggest coal mine in the Sindh Province of Pakistan [44]. Thar Coal is demarcated to be 175.5 billion tons of lignite or brown coal which can be utilized for the fuel and generation of electricity purpose. As shown in Figure 2, the Thar Coalfield is divided into 12 different blocks. Open-pit and underground mining methods can be used to extract coal from the area. Specifically, Block-II of the 12 blocks is fully developed for the open-pit mining method by Sindh Engro Coal Mining Company (SECMC), while some areas of Block-1 and BlockIX are being prepared for the underground mining method. The coal seam thickness of Block-IX at Thar Coalfield is about $12 \mathrm{~m}$ with an inclination of $0^{\circ}$ to $7^{\circ}$, and the topbottom plate is siltstone-claystone to claystone. However, for the first time in the history of Pakistan, Shahani et al. recommended the use of mechanized longwall top coal caving (LTCC) method in the Thar Coalfield, Pakistan $[44,45]$. At the same time, accurate determination of the mechanical properties of the Thar Coalfield, especially the UCS, plays an important role in providing a good understanding of the behavior of the roof and ground prior to mining operations. In this study, 106 samples of soft sedimentary rocks were initially collected randomly from BlockIX at Thar, as shown in Figure 2 of the yellow-colored area. The collected rock samples were then arranged and subdivided according to the recommended standards of the 
TABLE 1: Empirical equations proposed by the researchers for estimating UCS.

\begin{tabular}{lccccc}
\hline S. no & Country of origin & Rock type & No. of data & $R^{2}$ & References \\
\hline 1 & Turkey & Sedimentary & 18 & 0.82 & {$[7]$} \\
2 & USA & Mixed & 86 & 0.81 & {$[8]$} \\
3 & Spain & Mixed & 194 & 0.81 & {$[9]$} \\
4 & Japan and Indonesia & Mixed & 33 & 0.77 & [10] Based on part of dataset by [11] \\
5 & Turkey & Sedimentary & 150 & 0.65 & {$[12]$} \\
6 & India & Sedimentary & 13 & 0.87 & {$[13]$} \\
7 & Iran & Sedimentary & 40 & 0.94 & {$[14]$} \\
8 & Israel and USA & Mixed & 7 & 0.96 & {$[15]$} \\
9 & UK, France, and Denmark & Sedimentary & 7 & 0.9 & {$[16]$} \\
10 & England and Turkey & Mixed & 44 & 0.11 & {$[17]$} \\
11 & Greece & Metamorphic & 32 & 0.82 & {$[18]$} \\
12 & Spain & Sedimentary & 99 & 0.94 & {$[19]$} \\
13 & Turkey & Sedimentary & 19 & 0.78 & {$[20]$} \\
14 & Portugal & Igneous & 9 & 0.72 & {$[21]$} \\
15 & UK, France, and Denmark & Sedimentary & 7 & 0.87 & {$[16]$} \\
16 & United Kingdom & Igneous & 171 & 0.53 & {$[21]$} \\
\hline
\end{tabular}

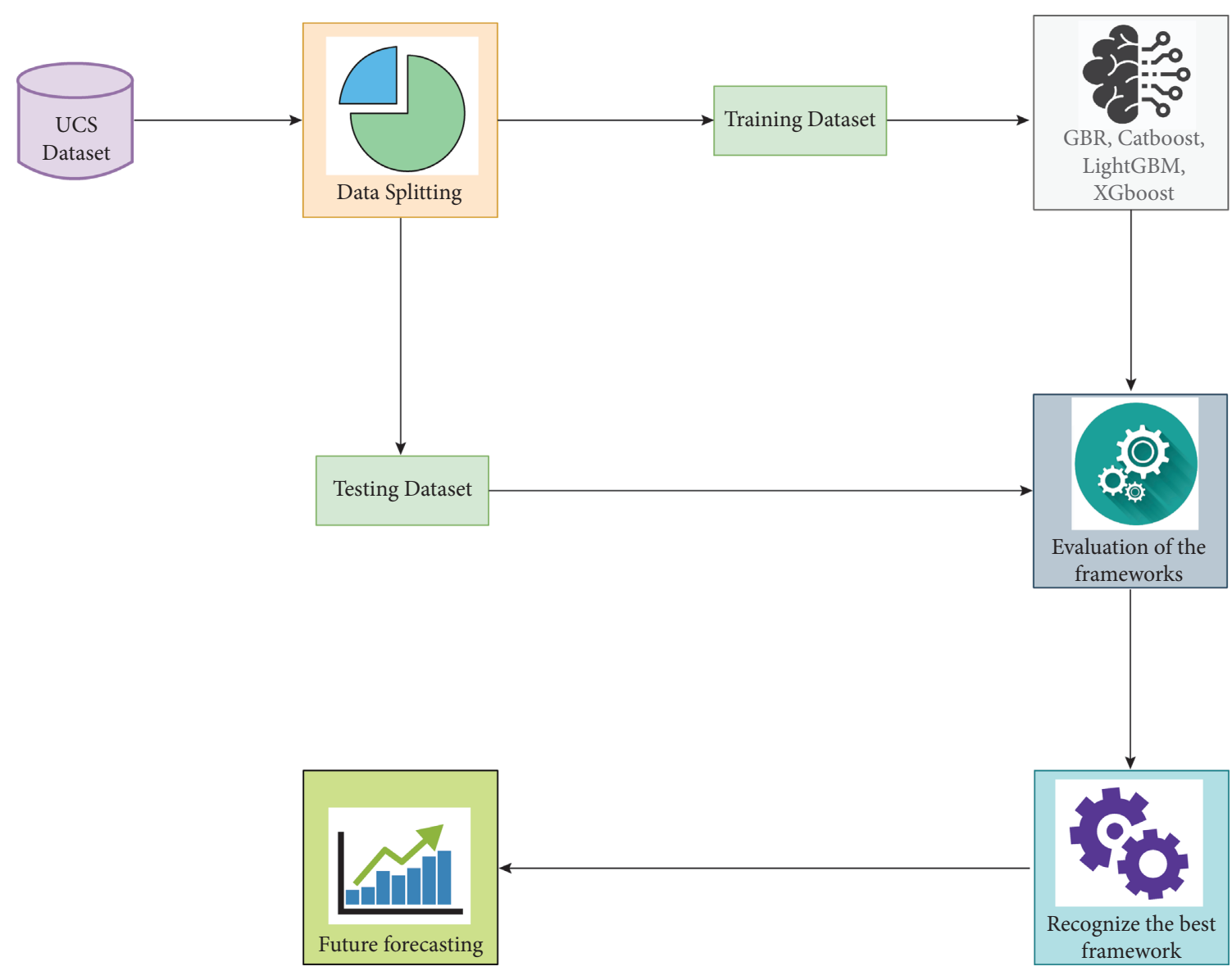

FIgURE 1: Flowchart of the study.

International Society of Rock Mechanics (ISRM) [46] and the American Society for Testing Methods (ASTM) [47] in order to maintain the same core size and geological and geometric characteristics. The experimental work on the studied rock samples was carried out in the laboratory of the Department of Mining Engineering, Mehran University of Engineering and Technology (MUET), to determine the physical and mechanical properties like $\rho_{\mathrm{w}}\left(\mathrm{g} / \mathrm{cm}^{3}\right)$; moisture $(\%) ; \rho_{\mathrm{d}}\left(\mathrm{g} / \mathrm{cm}^{3}\right)$; BTS $(\mathrm{MPa}) ;$ and UCS $(\mathrm{MPa})$. In Figure 3(a), a universal testing machine (UTM) was used to perform the UCS as shown in Figure 3(b) and a BTS as shown in Figure 3(c). Table 2 illustrates the entire dataset employed in the study. Table 3 denotes the statistical distribution of the UCS dataset. 


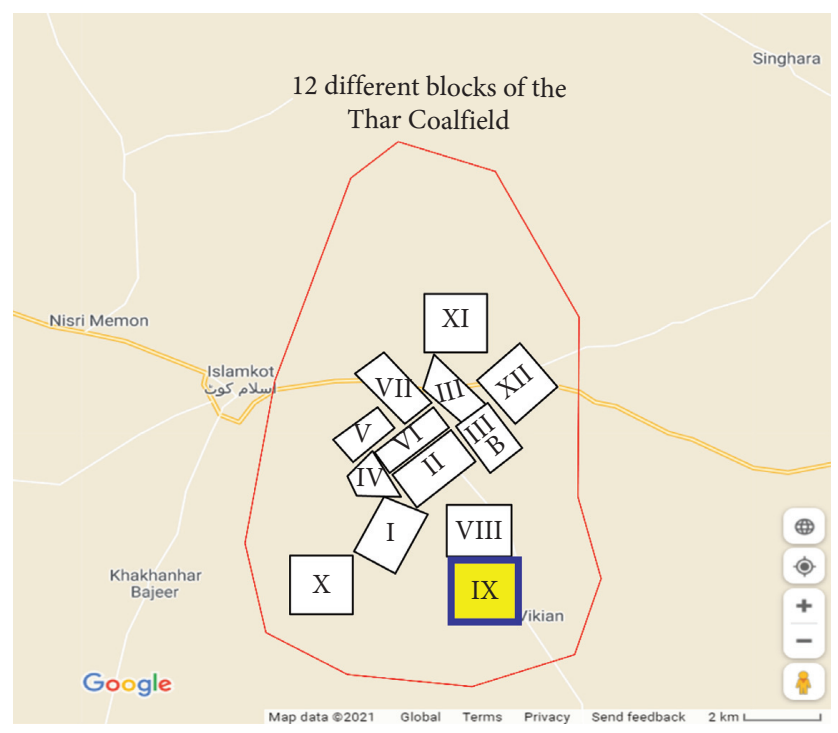

Figure 2: Location map of study area (Block-IX) of Thar Coalfield, Pakistan.

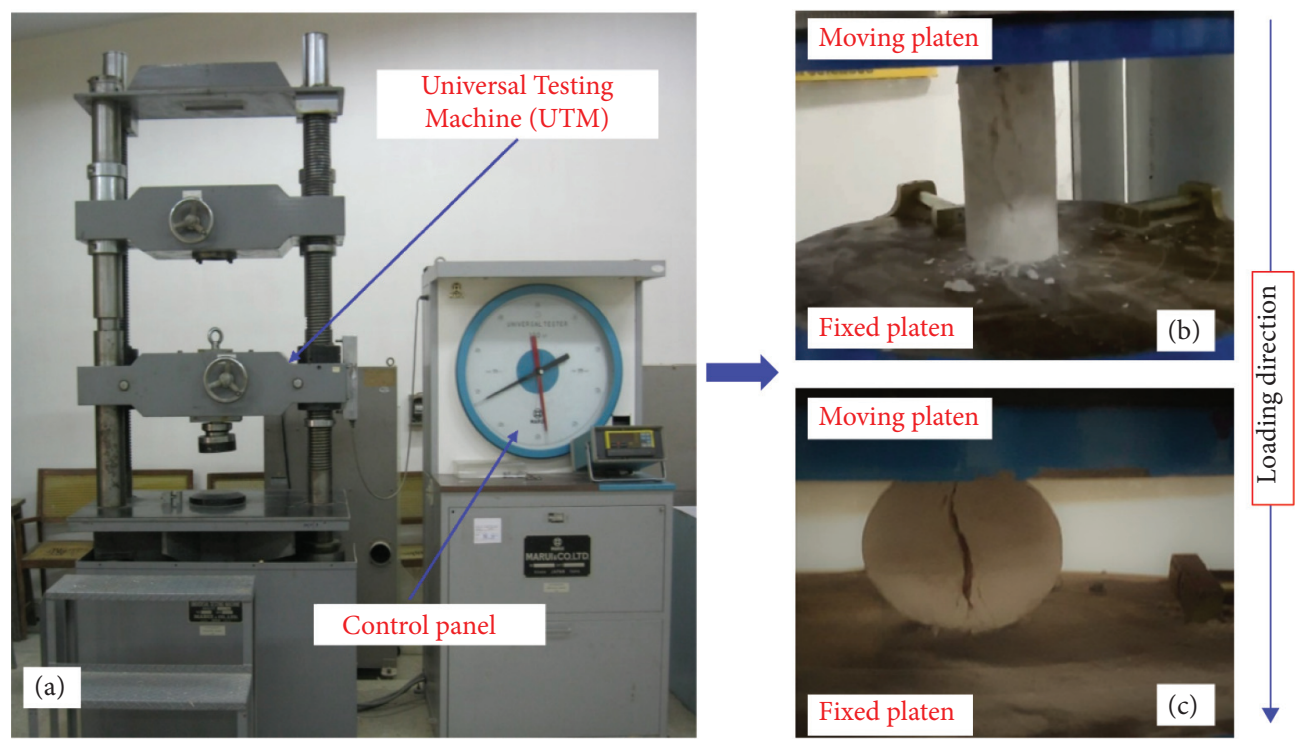

Figure 3: (a) Universal testing machine (UTM), (b) deformed rock core specimen for Brazilian tensile strength test, and (c) deformed rock core specimen for UCS test.

TABLE 2: Brief summary of UCS dataset.

\begin{tabular}{|c|c|c|c|c|c|}
\hline Serial no. & $\rho_{\mathrm{w}}\left(\mathrm{g} / \mathrm{cm}^{3}\right)$ & Moisture (\%) & $\rho_{\mathrm{d}}\left(\mathrm{g} / \mathrm{cm}^{3}\right)$ & BTS (MPa) & UCS (MPa) \\
\hline 1 & 2.132 & 11.78747 & 1.90719 & 0.305 & 0.404 \\
\hline 2 & 2.013 & 15.07577 & 1.749282 & 0.217402 & 0.491 \\
\hline 3 & 2.112 & 14.08254 & 1.851291 & 0.259 & 0.579 \\
\hline 4 & 2.119 & 17.0949 & 1.809643 & 0.394188 & 0.485 \\
\hline 5 & 2.034 & 15.59792 & 1.759547 & 0.336 & 0.52 \\
\hline$\ldots \ldots$ & $\ldots \ldots$ & $\ldots \ldots$ & $\ldots \ldots$ & $\cdots \cdots$ & $\cdots \cdots$ \\
\hline 102 & 2.003 & 20.67989 & 1.659763 & 0.502 & 3.35 \\
\hline 103 & 1.823 & 14.17769 & 1.596634 & 0.452715 & 2.46 \\
\hline 104 & 1.964 & 20.07648 & 1.635624 & 0.358732 & 3.448 \\
\hline 105 & 1.993 & 16.44345 & 1.71156 & 0.516271 & 3.549 \\
\hline 106 & 1.97 & 12.14405 & 1.756669 & 0.627239 & 3.28 \\
\hline
\end{tabular}


Figure 4 represents the pairwise distribution of various features and UCS. It can be seen that moisture and BTS are moderately correlated to the UCS whereas $\rho_{\mathrm{w}}$ and $\rho_{\mathrm{d}}$ are negatively correlated to the UCS. It is important to mention that each feature does not correlate well with UCS independently; hence all the features together are evaluated to predict the UCS.

\section{Methods}

3.1. Gradient Boosted Regressor (GBR). The gradient boosted regression (GBR) tree incorporates the weak learners; i.e., the learner algorithms moderately perform well as compared to random algorithms, into a strong learner in a repetitive technique [48]. In contrast to the bagging technique, the boosted mechanism produces the base frameworks consecutively. The robustness of the prediction model is enhanced by generating several frameworks in a series by focusing priority on this learning information that is complicated to evaluate. In the boosting mechanism, the previous base frameworks that are inappropriate to estimate are frequently developed in the training datasets compared to those models that are precisely evaluated. Each supplementary base framed is directed to rectify the errors produced by its preceding base framework. The existence of the boosting mechanism is from the response of Schapire to Keran's inquiry [49, 50] (Kearns): Is a combination of a weak learner an alternative to distinguish strong learner? Weak Learner is defined as the algorithm that is working well as compared to random approximation; a strong base framework is a more authentic classification or regression algorithm that is inconsistent that is efficiently corresponding with the problem. The response to such an inquiry is very significant. The evaluation of a weak framework is often unchallenging as compared with a strong framework. Schapire demonstrated that the response to Kearns' inquiry is "Yes" manifesting that many weak frameworks are combined into a high and single robust framework.

The main difference between boosted and bagging mechanism is that in boosting method the training datasets are systematically resampled in order to anticipate the most convenient instruction for each succeeding framework. The modified dissemination in each stage of training is contingent on the error generated by the preceding framework. In contrast, in the bagging mechanism, each trail is consistently designated to generate a training dataset, and the uncertainty of designating a single trail is divergent for the boosting mechanism. The trails that are incorrectly evaluated or misclassified have more probability to be set with higher weights. Hence, each freshly developed framework emphasizes the trail that has been incorrectly evaluated or misclassified by succeeding frameworks.

Boosting arranges the secondary frameworks that reduce a certain loss function averaged over the learning datasets, i.e., mean absolute error or squared mean error. The loss function calculates the aggregate of the forecasted value that varies from the measured value. A forward stage-wise modeling technique is one of the estimated solutions to the problem. This modeling technique consecutively adjoins new base frameworks without replacing the coefficient and parameter of the model that has previously been attached. With reference to the regression models, the boosting mechanism is a configuration of "functional gradient descent." Functional gradient descent is an optimization mechanism that reduces a certain loss function by attaching a base framework at each stage that minimizes well the loss function. Figure 5 shows the description of GBR employed in this study [51, 52].

Friedman suggested an improvement to the gradient boosted regression models by employing a predetermined amount of regression tree to the base framework. The modified framework enhances the performance of the Friedman model [53]. In order to predict the UCS of sedimentary rocks, the modified version of gradient boosted regression has been used. Table 4 illustrates the execution of a GBR framework in python. Consider that the leaves number for each tree is $l$, each tree splitting the input space into $l$ separate territory $T_{1 p}, T_{2 p} \ldots \ldots \ldots T_{\mathrm{lp}}$ and forecasting a constant value $k_{\mathrm{lp}}$ for territory $T_{\mathrm{lp}}$. The gradient boosting regression tree is given by

$$
f_{p}(a)=\sum_{l=1}^{L} k_{\mathrm{lp}} F\left(a \in T_{\mathrm{lp}}\right),
$$

where $F\left(a \in T_{\mathrm{lp}}\right)= \begin{cases}1, & \text { if } a \in T_{p} \\ 0, & \text { otherwise }\end{cases}$

By employing a regression tree to reinstate $f_{p}(a)$ in the generic gradient boosting mechanism, the framework gradient descent stage size and updating equation are given by equation (2) and (3), respectively.

$$
\begin{gathered}
f_{p}(a)=f_{p-1}(a)+\rho_{p} g_{p}(a) . \\
\rho_{p}=\operatorname{argmin}_{\rho} \sum_{l=1}^{L} M\left(b_{i}, f_{p-1}\left(a_{i}\right)+\rho g_{p}\left(a_{i}\right)\right) .
\end{gathered}
$$

Hence, equations (2) and (3) fit as equations (4) and (5):

$$
\begin{gathered}
f_{p}(a)=f_{p-1}(a)+\sum_{l=1}^{L} \rho_{p} k_{\mathrm{lp}} F\left(a \in T_{\mathrm{lp}}\right) . \\
\rho_{p}=\operatorname{argmin}_{\rho} \sum_{l=1}^{L} M\left(b_{i}, f_{p-1}\left(a_{i}\right)+\sum_{l=1}^{L} \rho_{p} k_{\mathrm{lp}} F\left(a \in T_{\mathrm{lp}}\right)\right) .
\end{gathered}
$$

By employing discrete ideal $\rho_{\mathrm{lp}}$ for each territory $T_{\mathrm{lp}} k_{\mathrm{lp}}$ are supposed to be detached. The updated framework equations (6) and (7) are given by

$$
\begin{gathered}
f_{p}(a)=f_{p-1}(a)+\sum_{l=1}^{L} \rho_{p} F\left(a \in T_{\mathrm{lp}}\right) . \\
\rho_{p}=\operatorname{argmin}_{\rho} \sum_{l=1}^{L} M\left(b_{i}, f_{p-1}\left(a_{i}\right)+\sum_{l=1}^{L} \rho_{p} F\left(a \in T_{l p}\right)\right) .
\end{gathered}
$$

The framework overfitting can be restrained through managing the gradient boosting repetitions number, or 
TABLE 3: Statistical distribution of UCS dataset.

\begin{tabular}{|c|c|c|c|c|c|}
\hline & $\rho_{\mathrm{w}}\left(\mathrm{g} / \mathrm{cm}^{3}\right)$ & Moisture (\%) & $\rho_{\mathrm{d}}\left(\mathrm{g} / \mathrm{cm}^{3}\right)$ & $\mathrm{BTS}(\mathrm{MPa})$ & UCS (MPa) \\
\hline Mean & 1.93 & 18.28 & 1.66 & 0.29 & 1.31 \\
\hline Standard deviation & 0.37 & 8.93 & 0.39 & 0.12 & 0.93 \\
\hline Minimum & 1.005 & 6.63 & 0.76 & 0.02 & 0.26 \\
\hline Maximum & 2.38 & 43.3 & 2.15 & 0.62 & 3.623 \\
\hline
\end{tabular}

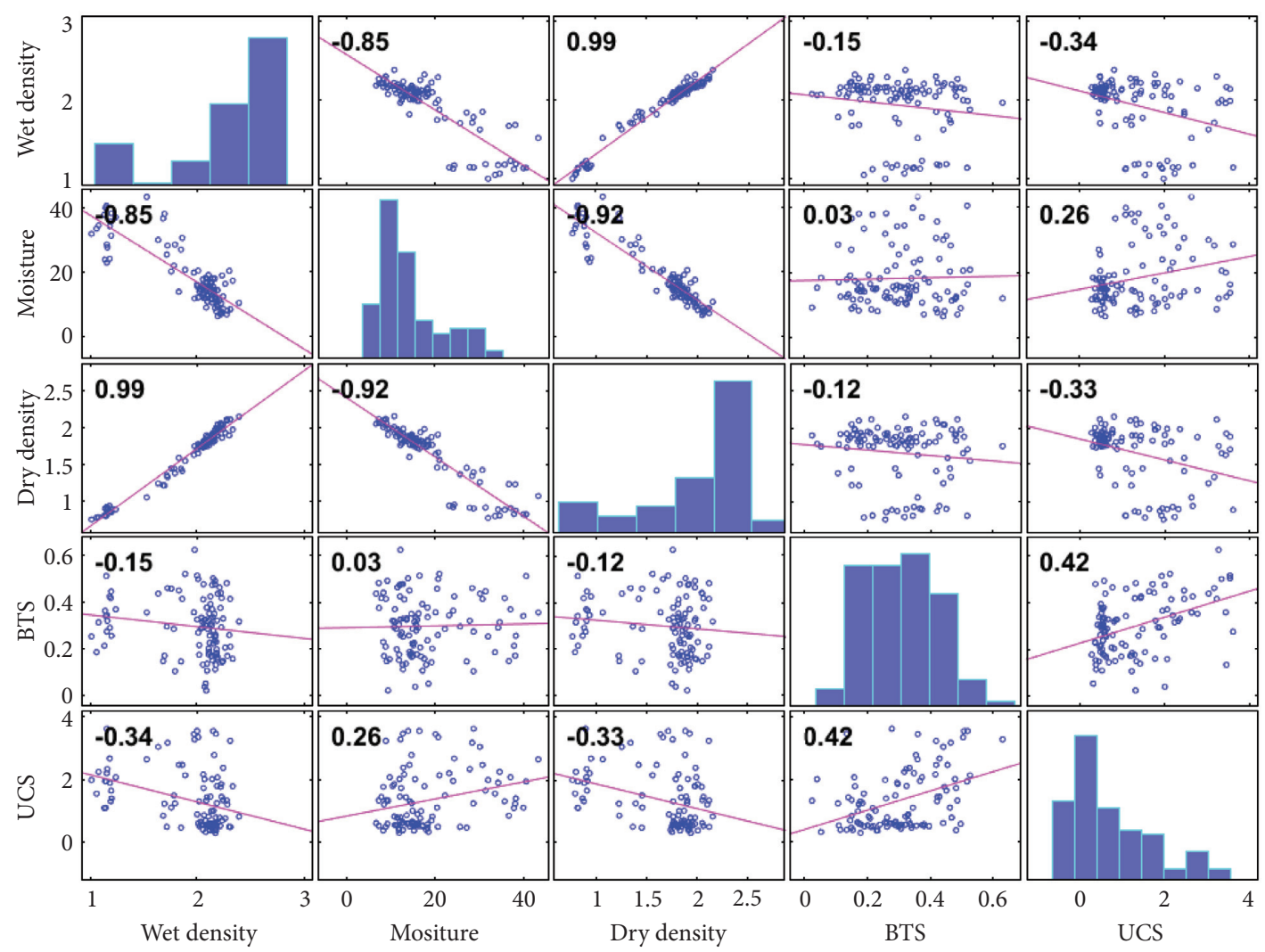

FIgURE 4: Pairwise correlation between the input variables and output in the original dataset.

more competent, appraising the beneficiation of each tree by $J \in(0,1)$. Hence, the updated model is given by

$$
f_{p}(a)=f_{p-1}(a)+L \sum_{l=1}^{L} \rho_{p} F\left(a \in T_{\mathrm{lp}}\right) \text {. }
$$

3.2. Catboost. Catboost is a type of gradient boosting algorithm that is succeeded in recent times by Dorogush et al. [54]. Catboost tackles both the regression and classification problems and has been publicized in a new free open-source multiplatform gradient boosting library [54, 55]. Decision trees are employed as a base weak learner in the Catboost algorithm and gradient boosting to sequentially fit on the decision tree. In order to enhance the execution of the Catboost algorithm and to avoid overfitting, the inconsistent permutation of the learning information of the gradient is employed [54].

The Catboost algorithm aims to minimize the forecasting relocation that happens during the learning stage. The dissemination relocation is the removal of $\mathbf{F}(\mathbf{y}) \mid\left(y_{i}\right)$ with $y_{i}$ being a learning sample, with relation to $\mathbf{F}(\mathbf{y}) \mid\left(y_{i}\right)$ for a test sample $y$. At the learning stage, gradient boosting employs the same sample for the calculation of gradient and the framework that reduces that gradient. The concept of Catboost is to establish j...n, the base framework for individual $P$ boosting repetition. The ith framework of the $m$ th repetition is learnt of initial ith samples of the permutation and is applicable to calculate the gradient of the $j+1$ sample for $p+1$ repetition. Succeeding, to be unrestrained of the start arbitrary permutation, this technique employs $s$ reciprocated arbitrary permutation. A distinguish framework is constructed per repetition that manages all permutations and frameworks. Symmetric trees work as a foundation for the frameworks. The trees are extended by growing all the leaf nodes level-wise by using the same splitting criteria.

The mechanism introduced in the Catboost algorithm is to calculate the contemporary character that is identical to the one imitated for establishing the network. Thus, for a specified arbitrary permutation of the samples, the data sample $<i$ is used to calculating the character value for each 


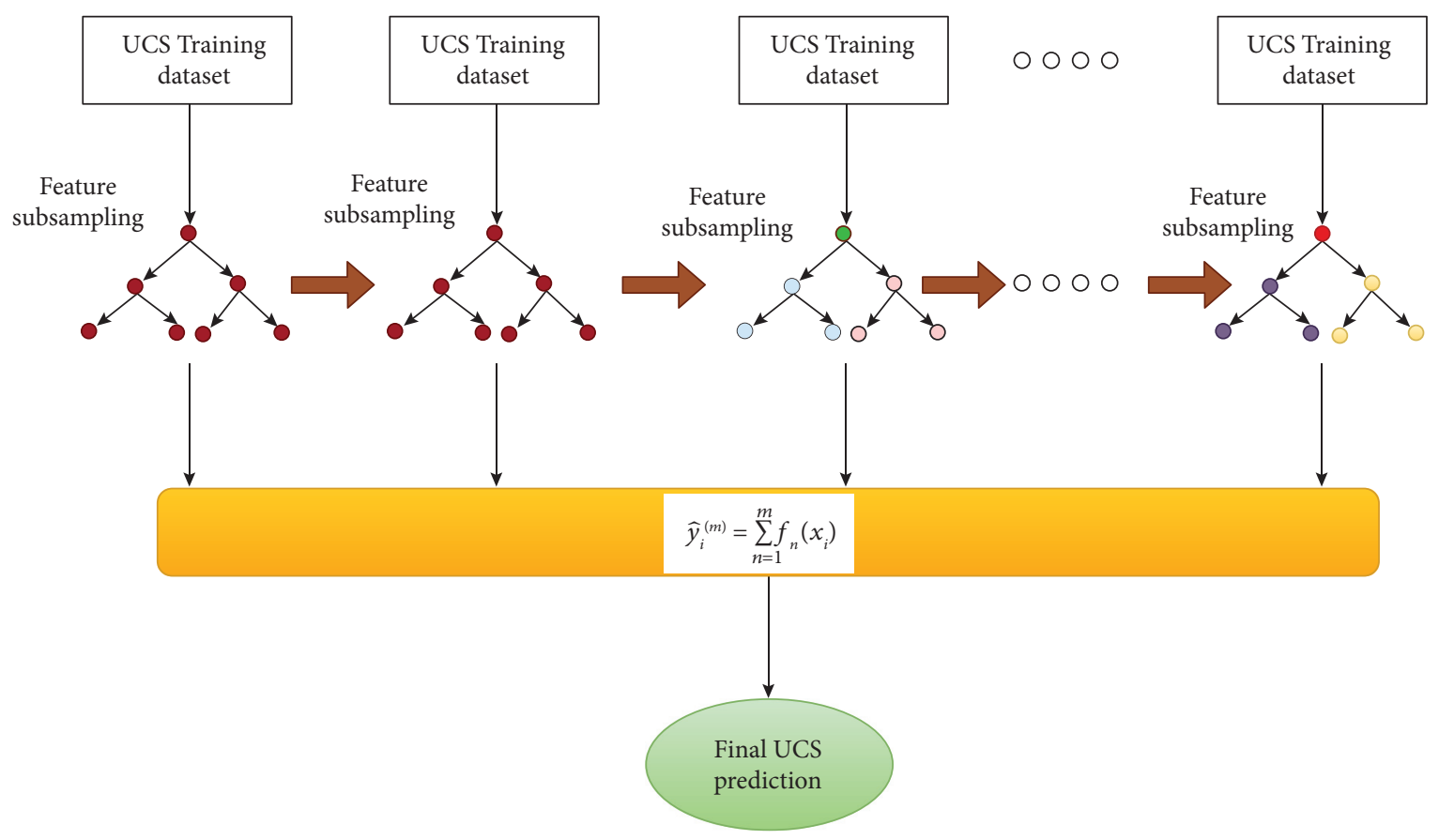

Figure 5: Description of GBR employed in this study.

sample $i$. Later on, various permutations are executed and the acquired character value for each sample is averaged. Catboost is a large-scale and comprehensive library that is composed of various elements as standard boosting, GPU learning and consists of tenfold hyperparameters optimization to modify to several practicable investigation circumstances. Standard gradient boosting is also a part of the Catboost algorithm. Figure 6 depicts the description of Catboost employed in this study.

It should be noted that the training capability of the Catboost algorithm is supervised by its framework hypermeter, namely, the number of iterations, learning rate, maximum depth, etc. The determination of the best hyperparameters for a model is a difficult, time-consuming, challenging, and tedious task contingent on the user's skills and expertise. Table 5 illustrates the execution of a Catboost framework in python employed in this study.

\subsection{Light Gradient Boosting Machine (LightGBM). Light} Gradient Boosting Machine, abbreviated as LightGBM, is an open-source gradient boosting machine learning framework by Microsoft that uses a decision tree as a based training algorithm [56]. LightGBM inserts consecutive element value buckets into discrete bins with higher efficiency and faster training speed. It employs the histogram-based algorithms $[57,58]$ in order to enhance the learning stage, minimize memory consumption, and incorporate an updated communication network to improve training uniformity, known as parallel voting decision tree machine learning algorithm. The learning information is split into multiple trees, the local voting technique is implemented to choose the top-k element, and the globing voting technique is acquired in each repetition. LightGBM manipulates the leaf-wise strategy to perceive a leaf with maximum splitter gain as shown in Figure 7. It can be used for classification, regression, sorting, and numerous machine learning projects. LightGBM builds a sophisticated tree compared with the level-wise distribution technique by the leaf-wise distribution technique, which is the principal component for executing the algorithm with higher efficiency. Even so, it gives rise to overfitting; however, it can be prohibited by using the max-depth element in LightGBM.

LightGBM [56] is a comprehensive library that executes gradient boosting and intends some modification. The execution of gradient boosting has been mainly concentrated on building a computationally systematic algorithm. The library comprises tenfold training hyperparameters that authenticate this framework to implement in the diverse scenario. LightGBM execution also suggests advanced features both in CPU and in GPU; it can operate as gradient boosting and has multifold combinations including bootstrap subsampling and column randomization. LightGBM is broadly featured by Gradient-based One-Side Sampling and Exclusive Feature Bundling. Gradient-based One-Side Sampling is a subsample mechanism employed to build the learning information as a base tree in the ensemble. In the Adaboost machine learning algorithm, the objective of this mechanism is to enhance the importance of the sample with a higher probability, which is associated with the sample having a higher gradient. When the Gradient-based OneSide Sampling is implemented, the learning information for the base learner is formulated on the top fraction of the sample with a higher gradient (a) plus an arbitrary instance fraction (b) reacquired from the samples with a smaller gradient. To remunerate for the variation of measured dissemination, the sample in the lower gradient category is arranged together and weighed up by $(1-x) / y$ when 
TABLE 4: Executing a GBR framework in python employing the gradient boost regressor.

\begin{tabular}{|c|c|}
\hline Description & Python script \\
\hline \multirow[t]{5}{*}{ Import the python packages } & $\begin{array}{l}\text { from sklearn.ensemble import GradientBoostingRegressor from sklearn.model_selection import } \\
\text { cross_val_score, RepeatedKFold }\end{array}$ \\
\hline & Framework $=$ GradientBoostingRegressor () \\
\hline & \# Interpretation of the evaluation mechanism \\
\hline & $\mathrm{cv}=$ RepeatedKFold $\left(\mathrm{n} \_\right.$splits $=10, \mathrm{n} \_$repeats $=3$, random_state $\left.=1\right)$ \\
\hline & \# Framework assessment \\
\hline \multirow[t]{5}{*}{$\begin{array}{l}\text { Interpretation of the framework } \\
\text { attributes }\end{array}$} & $\begin{array}{l}\text { n_scores }=\text { cross_val_score }(\text { Framework, } \mathrm{x} \text { _train, UCS_train, scoring = 'neg_mean_absolute_error', } \\
\mathrm{cv}=\mathrm{cv}, \mathrm{n} \text { _jobs }=-1)\end{array}$ \\
\hline & \# Performance Report \\
\hline & print('MAE: \%.8f (\%.8f)' \% (mean(n_scores), std(n_scores))) \\
\hline & \# fit the framework on training dataset \\
\hline & Framework.fit(x, UCS) \\
\hline Training dataset & Train_dataset_Forecasting = Framework.predict(x_train) \\
\hline Testing dataset & Test_dataset_Forecasting = Framework.predict(x_test) \\
\hline
\end{tabular}

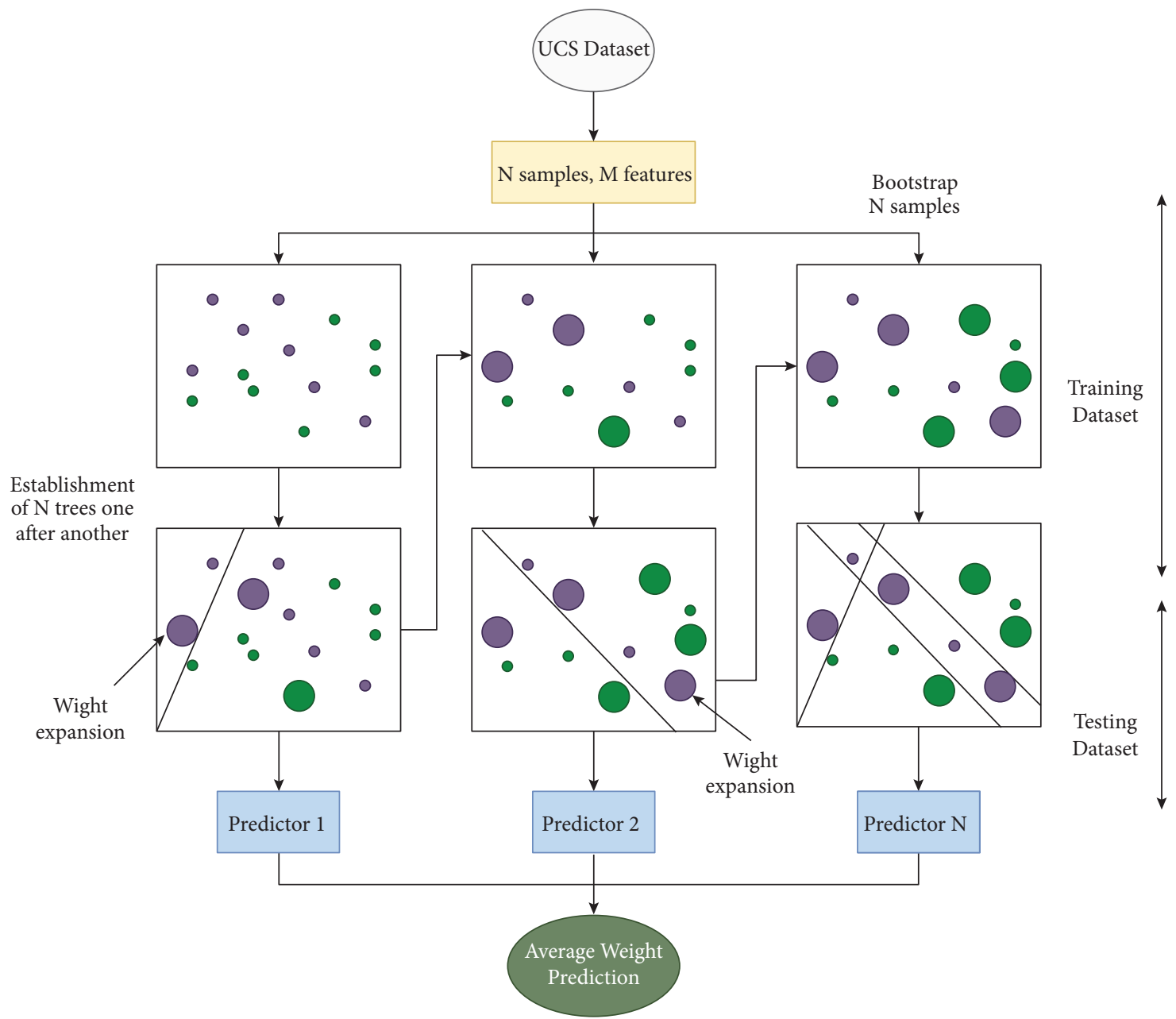

Figure 6: Description of Catboost employed in this study.

calculating the data gain. On the other hand, the Exclusive Feature Bundling mechanism accumulates the sparse element into a single element. This can be accomplished without preventing any knowledge when those elements do not consist of a nonzero number coincidently. The mechanism anticipates supplementary learning rate gain. Table 6 illustrates the execution of a LightGBM framework in python employed in this study.

3.4. Extreme Gradient Boosting (XGBoost). Extreme gradient boosting (XGBoost) is one of the significant types of 
TABLE 5: Executing a catboost framework in python employing the catboost regressor.

\begin{tabular}{|c|c|}
\hline Description & Python script \\
\hline \multirow{2}{*}{ Import the python packages } & from catboost import CatBoostRegressor \\
\hline & from sklearn.model_selection import RepeatedKFold, cross_val_score \\
\hline \multirow{12}{*}{$\begin{array}{l}\text { Interpretation of the framework } \\
\text { attributes }\end{array}$} & Framework = CatBoostRegressor $\left(\right.$ verbose $=0, \mathrm{n} \_$estimators $\left.=100\right)$ \\
\hline & \# Interpretation of the evaluation Mechanism \\
\hline & $\mathrm{cv}=$ RepeatedKFold $\left(\mathrm{n} \_\right.$splits $=10, \mathrm{n} \_$repeats $=3$, random_state $\left.=1\right)$ \\
\hline & \# Framework assessment \\
\hline & n_scores $=$ cross_val_score(Framework, x_train, UCS_train, \\
\hline & scoring = 'neg_mean_absolute_error', \\
\hline & $\mathrm{cv}=\mathrm{cv}, \mathrm{n} \_$jobs $=-1$, error_score $=$ 'raise') \\
\hline & \# Performance Report \\
\hline & print('MAE: \%.8f (\%.8f)' \% (mean(n_scores), std(n_scores))) \\
\hline & \# fit the framework on training dataset \\
\hline & Framework $=$ CatBoostRegressor $\left(\right.$ verbose $=0, \mathrm{n} \_$estimators $\left.=100\right)$ \\
\hline & Framework.fit(x, UCS) \\
\hline Training dataset & train_dataset_Forecasting = Framework.predict(x_train) \\
\hline Testing dataset & test_dataset_Forecasting = Framework.predict(x_test) \\
\hline
\end{tabular}

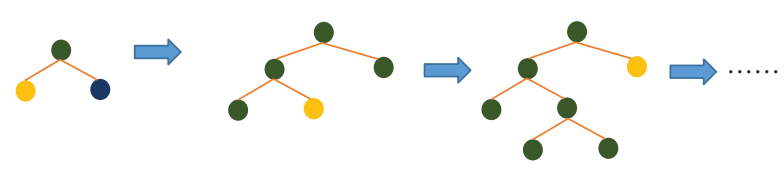

FIGURE 7: Description of LightGBM employed in this study.

ensemble learning algorithms in machine learning methods [59]. It comprises ordinary regression and classification trees by incorporating analytical boosting techniques. Boosting enhances the precision estimation of the framework by building various trees as a substitution to establish a targeted tree and later on adding them to determine a coherent forecasting model [60]. XGBoost initiates the tree by sequentially retaining the residuals of past trees as influences on the consequence tree. Due to this, the consequence tree establishes comprehensive forecasting by generating the error of past trees. At the stage when the loss function is reduced, the sequential framework structure relation can be segmented as a gradient descent type that improves the prediction by adding an extra tree at each step to lower the reduction [61]. Tree growth ends when a preestablished number of the most extraordinary tree is gained, or when the error of the training stage cannot be augmented to a predicted sequential tree number. The performance promptness and evaluation accuracy of the gradient boosting can be significantly increased by attaching arbitrary investigation. Particularly for each tree in a symmetry, an arbitrary training data subsample is considered from the entire training dataset, excluding the replacement. Instead of the entire sample, this arbitrarily described subsample is then utilized to fit the tree and is ascertained to update the framework. XGBoost is a state-of-the-art redistributed gradient boosting algorithm that can manage and achieve up-to-date forecasting demonstration [62]. The second-order evaluation of the loss function is employed in the XGBoost algorithm which is expeditious and speedy as compared to the conventual gradient boosting algorithms. It has been extensively adopted to mine gene coupling features. Figure 8 depicts the description of XGBoost employed in this study.

Consider $\overline{u_{i}}$ is the forecasted result of $i$ th number of data for which the characteristics vector is $V_{i} ; E$ shows the estimators number for each estimator $f_{k}$ (with $k$ from 1 to E) corresponding to individual tree anatomy, and $u_{i}^{0}$ depicts the preliminary assumption that is the average of the measured characteristics in the learning information. The following equation implements various expansion functions to forecast the results:

$$
\overline{u_{i}}=u_{i}^{0}+\eta \sum_{K=1}^{E} f_{k}\left(V_{i}\right)
$$

whereas the $\eta$ parameter is the learning rate that is adjoined to improve the model implementation to enhance the model, to execute rhythmically while linking the latest tree and confront overfitting.

Concerning Eq. 9, at kth state, a character kth is connected to the model and the kth forecasted value $u_{i}^{-k}$ is implemented from the forecasted value at the preceding state $u_{i}^{-(k-1)}$ and the augmented $f_{k}$ of the character of the attached $k$ th character is depicted in

$$
u_{i}^{-k}=u_{i}^{-(k-1)}+\eta f_{k},
$$

whereas $f_{k}$ shows the weight of leaves that are developed by reducing the objective function of the kth tree represented by

$$
\text { obj }=\gamma N+\sum_{a=1}^{N}\left[G_{a} \omega_{a}+\frac{1}{2}\left(H_{a}+\lambda\right) \omega_{a}^{2},\right.
$$


TABLE 6: Executing a lightgbm algorithm in python employing the lightgbm regressor.

\begin{tabular}{|c|c|}
\hline Description & Python script \\
\hline \multirow[t]{5}{*}{ Import the python packages } & $\begin{array}{l}\text { from lightgbm import LGBMRegressor } \\
\text { from sklearn.model_selection import cross_val_score, RepeatedKFold }\end{array}$ \\
\hline & Framework $=$ LGBMRegressor () \\
\hline & \# Interpretation of the evaluation Mechanism \\
\hline & $\mathrm{cv}=$ RepeatedKFold $\left(\mathrm{n} \_\right.$splits $=10, \mathrm{n} \_$repeats $=3$, random_state $\left.=1\right)$ \\
\hline & \# Framework assessment \\
\hline $\begin{array}{l}\text { Interpretation of the framework } \\
\text { attributes }\end{array}$ & $\begin{aligned} \text { n_scores }= & \text { cross_val_score }\left(\text { Framework, } x \_t r a i n, \text { UCS_train, }\right. \\
& \text { scoring }=\text { 'neg_mean_absolute_error', } \\
& \text { cv }=\text { cv, n_jobs }=-1, \text { error_score }=\text { 'raise' })\end{aligned}$ \\
\hline & \# Performance Report \\
\hline & print('MAE: $\% .8 \mathrm{f}(\% .8 \mathrm{f})$ ' $\%$ (mean(n_scores), std(n_scores))) \\
\hline & \# fit the framework on training dataset \\
\hline & $\begin{array}{l}\text { Framework.fit(x, UCS) } \\
\end{array}$ \\
\hline Training dataset & train_dataset_Forecasting = Framework.predict(X_train) \\
\hline Testing dataset & test_dataset_Forecasting = Framework.predict(X_test) \\
\hline
\end{tabular}

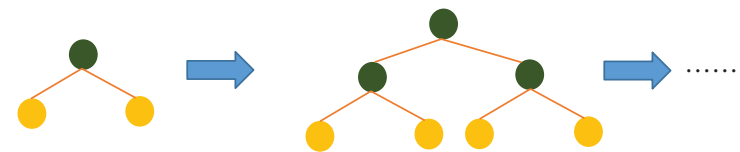

FIGURE 8: Description of XGBoost employed in this study.

whereas $\mathrm{N}$ designates the leaves of the kth tree and $\omega_{a}$ represent the weight of the leaves from 1 to $\mathrm{N}$, and $\gamma$ and $\lambda$ are the uniformity features that are utilized to implement the coherence to the anatomy to evade the overfitting in the model. The parameters $H_{a}$ and $G_{a}$ are the aggregate of entire information attached with data $a$ leaf of the prior and subsequent loss function gradient, respectively.

In sequence to build the kth tree, a single leaf is split into different numeration leaves. Eq. 12 indicates the anatomy of utilizing the gain parameters. Consider $C_{R}$ and $D_{R}$ depict the interdependent right leaf, and $C_{L}$ and $D_{L}$ are interdependent left leaf attaining the divergence. At the time, the gain parameter is close by zero, and the diverging benchmark is traditionally assumed. The $\gamma$ and $\lambda$ are the uniformity features that are periphrastically susceptible on the gain feature; i.e., higher the regularization parameter will decrease the gain parameter which in result will avoid the convolution of the excruciating of the leaf. But it will decrease the ability of the framework in order to apt to the training dataset.

$$
\text { gain }=\frac{1}{2}\left[\frac{C_{L}^{2}}{D_{L}+\lambda}+\frac{C_{R}^{2}}{D_{R}+\lambda}+\frac{\left(C_{L}+C_{R}\right)^{2}}{D_{L}+D_{R}+\lambda}\right] \text {. }
$$

XGBoost is a widely used machine algorithm that assembles an articulated and logical accomplishment of gradient boosting machine learning algorithm. A numerical value is the outcome of the regression prediction model problems. XGBoost can be practiced promptly to probabilistic regression frameworks. The ensembles are established from the decision tree model. Ensembles successively link trees and adjust the forecasting imprecision models. These types of ensemble machine learning techniques are known as boosting. The frameworks are established by implementing any arbitrary gradient descent optimization technique and distinct loss function. When the model is implemented, the gradient loss function is decreased, and therefore this mechanism is recognized as "gradient boosting." Table 7 illustrates the execution of an XGBoost framework in python employed in this study. XGBoost framework performed well on the UCS datasets as compared to GBR, Catboost, and LightGBM with the same parameter $\mathrm{n}_{-}$splits $=10$, $\mathrm{n} \_$repeats $=3$, random_state $=1$ (all the other parameters were used as a default in python). However, in order to further enhance the performance of the XGBoost framework, gbm_param_grid was further implemented in this study.

3.5. Performance Indices. In order to accurately and approximately assess the performance of machine learning algorithms, various researchers have employed different evaluation criteria, i.e., coefficient of correlation $\left(R^{2}\right)$ [63], mean absolute error (MAE), mean square error (MSE) [64], and root mean square error (RMSE) [65]. The higher value of $R^{2}$ and the lower MAE, MSE, and RMSE indicate that the predicted target values perform better in each proposed model. In this study, four estimation criteria are employed to narrate the association between measured and predicted UCS values, i.e., $R^{2}$, MAE, MSE, and RMSE as shown in Table 8.

\section{Results and Discussion}

UCS plays a significant role in the stability of geostructures. In this study, the use of novel machine learning methods to estimate the accuracy of the UCS is necessary. This is because the accuracy of the studied UCS can help in designing any type of rock engineering project. Therefore, this study aims to incorporate these gradient boosting machine learning algorithms, namely, GBR, Catboost, LightGBM, and XGBoost to predict the UCS of soft sedimentary rocks of Block-IX at Thar Coalfield, Pakistan, by using four input variables, i.e., $\rho_{\mathrm{w}}\left(\mathrm{g} / \mathrm{cm}^{3}\right)$; moisture (\%); $\rho_{\mathrm{d}}\left(\mathrm{g} / \mathrm{cm}^{3}\right)$; and BTS $(\mathrm{MPa})$. Then, the 106-point dataset for each model was identically assigned as $70 \%$ for the training phase to train the model and 30\% for the testing phase to test the model. In the end, the ultimate output of predicted UCS is as follows. 
TABLE 7: Executing a xgb framework in python employing the XGBoost.

Description

Import the python packages

Interpretation of the framework features

Create the parameter grid

Training dataset

Testing dataset

\section{Python script}

import xgboost as xgb

from xgboost import XGBRegressor

from sklearn.model_selection import train_test_split, cross_val_score, RepeatedKFold

from sklearn.metrics import mean_squared_error as MSE

Framework = XGBRegressor $($ objective $=$ 'reg:squarederror')

\# Interpretation of the evaluation Mechanism

$\mathrm{cv}=\operatorname{RepeatedKFold}\left(\mathrm{n} \_\right.$splits $=10, \mathrm{n} \_$repeats $=3$, random_state $\left.=1\right)$

\# Framework assessment

n_scores $=$ cross_val_score $\left(\right.$ Framework, $\left.x \_t r a i n, U C S \_t r a i n\right)$

print('MAE: \%.8f (\%.8f)' \% (mean(n_scores), std(n_scores))

\# fit the framework on training dataset

Framework $=$ XGBRegressor $($ objective $=$ 'reg:squarederror' $)$

Framework.fit(x, UCS)

gbm_param_grid $=\{$

"eta": $[0.2,0.25,0.3,0.35,0.4,0.5,0.65,0.7,0.8,0.9]$,

"max_depth": $[1,2,3,4,5,6,7,8,9,10]$,

"colsample_bytree": [0.5,0.55,0.6,0.62,0.65,0.7,0.8,0.9,0.95,1],

"subsample": [0.5,0.55,0.6,0.62,0.65,0.7,0.8,0.9,0.95,1],

\}

\# Instantiate the regressor: gbm

$\mathrm{gbm}=\mathrm{xgb}$. XGBRegressor $($ objective $=$ 'reg:squarederror')

\# Implement grid search: grid_mse

randomized_mse $=$ RandomizedSearchCV ( estimator $=\mathrm{gbm}$,

param_distributions $=$ gbm_param_grid,

scoring = "neg_mean_squared_error",

$\mathrm{cv}=3$,

verbose $=1$,

)

\# Fit grid_mse to the UCS training dataset

randomized_mse.fit(x, UCS)

train_dataset_forecasting $=$ randomized_mse.predict(x_train)

test_dataset_forecasting = randomized_mse.predict(x_test)
Figures 9(a) to 9(d) and 10(a) to 10(d) show the scatter plots of the predicted UCS as opposed to the measured UCS in the training and testing phases, respectively, employing various algorithms. In Figures 9(a) to 9(d), the correlation coefficient $\left(R^{2}\right)$ values of the GBR, Catboost, LightGBM, and XGBoost are 0.97, 0.95, 0.40, and 0.99 in the training phase, respectively. At the same time, Figures 10 (a) to 10 (d) demonstrate the $R^{2}$ values of the GBR, Catboost, LightGBM, and XGBoost are 0.94, 0.91, 0.39, and 0.99 in the testing phase, respectively. Figures 11 and 12 reveal the performance plots of predicted UCS as opposed to measured UCS in the training phase. Figure 12 reveals the performance plots of predicted UCS as opposed to measured UCS in the testing phase.

Figure 13 shows the various performance indices of proposed algorithms such as $R^{2}$, MAE, MSE, and RMSE. The performance indices can tell us the evaluation of the developed model by which we can distinguish the most suitable model. In this study, the performance indices of the proposed algorithms are tabulated in Table 9.

Taylor diagram describes a concise statistical description of how well the frameworks match their standard deviation and correlation. The following equation [66] represents the formula of the Taylor diagram:

$$
R=\frac{1 / P \sum_{P}^{P}\left(r_{n}-\bar{r}\right)\left(f_{n}-\bar{f}\right)}{\sigma_{r} \sigma_{f}},
$$

where $R$ represents a correlation, $P$ is the discrete point number, $r_{n}$ and $f_{n}$ are two vectors, $\sigma_{r}$ and $\sigma_{f}$ show the standard deviation of $r$ and $f$, and $r_{n}$ and $f_{n}$ depict the average value of vectors $r_{n}$ and $f_{n}$, respectively.

Figure 14 shows the association among the standard deviation, RMSE, and correlation of predicted UCS and measured UCS of GBR, Catboost, LightGBM, and XGBoost algorithms, respectively, from Figure 13. According to the results, the UCS of the XGBoost prediction algorithm was highly correlated with the measured UCS compared to the other studied algorithms. In addition, the standard deviation of the XGBoost is nearest to the original value of the standard deviation. As a result, the XGBoost demonstrates high accuracy as compared to the existing publicly available literature [7, 21, 40], and is proved to be the high accurate algorithm among all for UCS prediction. The GBR also has a standard deviation nearer to the original standard deviation but shows the smallest $R^{2}$ value. Meantime, Catboost and LightGBM have the smallest correlation and are further away from the original standard deviation values. 
TABle 8: Performance indices employed in this work.

\begin{tabular}{|c|c|c|}
\hline $\begin{array}{l}\text { Performance } \\
\text { indices }\end{array}$ & Definition & Mathematical formula \\
\hline$R^{2}$ & $\begin{array}{l}R^{2} \text { is an aggregate bench used to compute } \\
\text { how significant the association between } \\
\text { measured and predicted UCS values is. }\end{array}$ & $R^{2}=1-\sum_{i=1}^{n}\left(\mathrm{UCS}_{\text {measured }}-\mathrm{UCS}_{\text {predicted }}\right)^{2} / \sum_{i=1}^{n}\left(\mathrm{UCS}_{\text {measured }}-\mathrm{UCS}_{\text {mean }}\right)^{2}$ \\
\hline MAE & $\begin{array}{l}\text { MAE shows the mean absolute error, which } \\
\text { is a conventionally employed statistical } \\
\text { feature that can inspect the real scenario of } \\
\text { the predicted UCS value error. }\end{array}$ & $\mathrm{MAE}=\sum_{i=1}^{n}\left|\mathrm{UCS}_{\text {mean }}-\mathrm{UCS}_{\text {measured }}\right|$ \\
\hline MSE & $\begin{array}{l}\text { MSE as shown by equation (15) is a broadly } \\
\text { practiced mathematical standard that shows } \\
\text { the appropriate standard deviation of the } \\
\text { error relative to the measured and } \\
\text { predictive UCS value. }\end{array}$ & $\mathrm{MSE}=1 / T \sum_{i=1}^{n}\left(\mathrm{UCS}_{\text {measured }}-\mathrm{UCS}_{\text {mean }}\right)^{2}$ \\
\hline RMSE & $\begin{array}{l}\text { RMSE is a statistical measure used to } \\
\text { compute the mean of square error in UCS } \\
\text { database. }\end{array}$ & $\mathrm{RMSE}=\sqrt{1 / 2 \sum_{i=1}^{n}\left(\mathrm{UCS}_{\text {measured }}-\mathrm{UCS}_{\text {mean }}\right)^{2}}$ \\
\hline
\end{tabular}

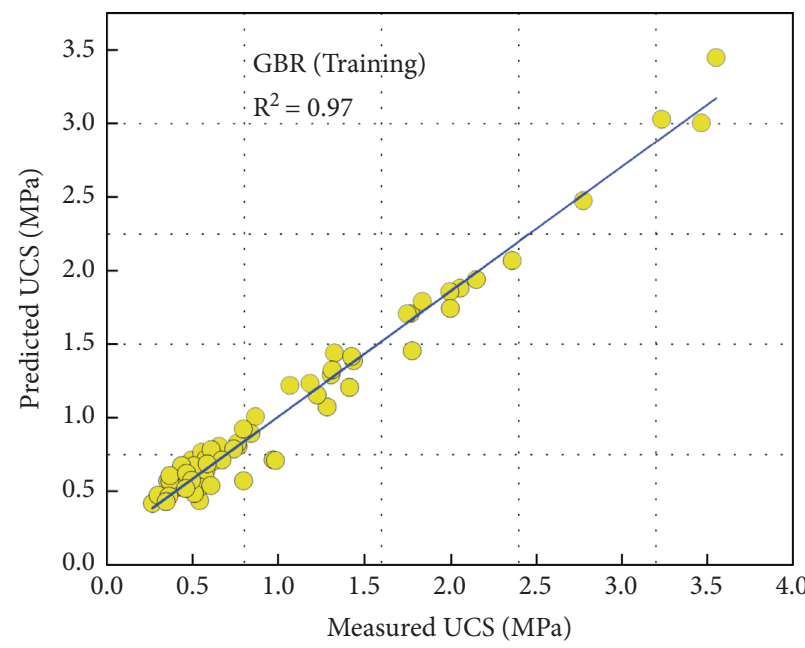

(a)

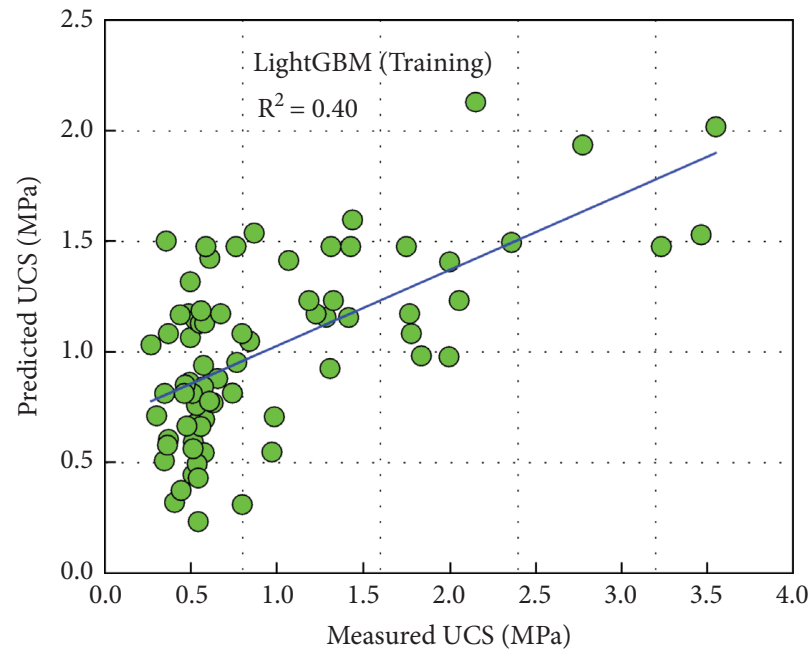

(c)

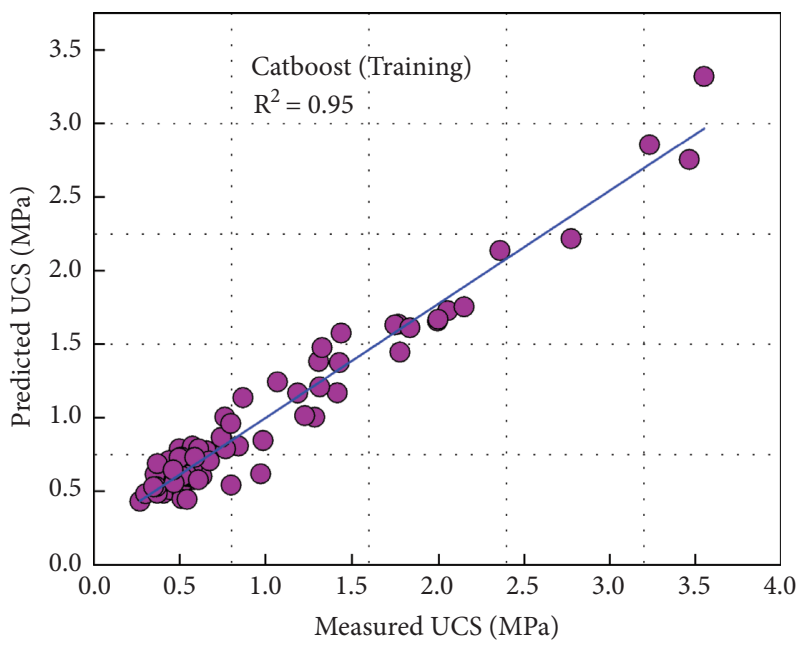

(b)

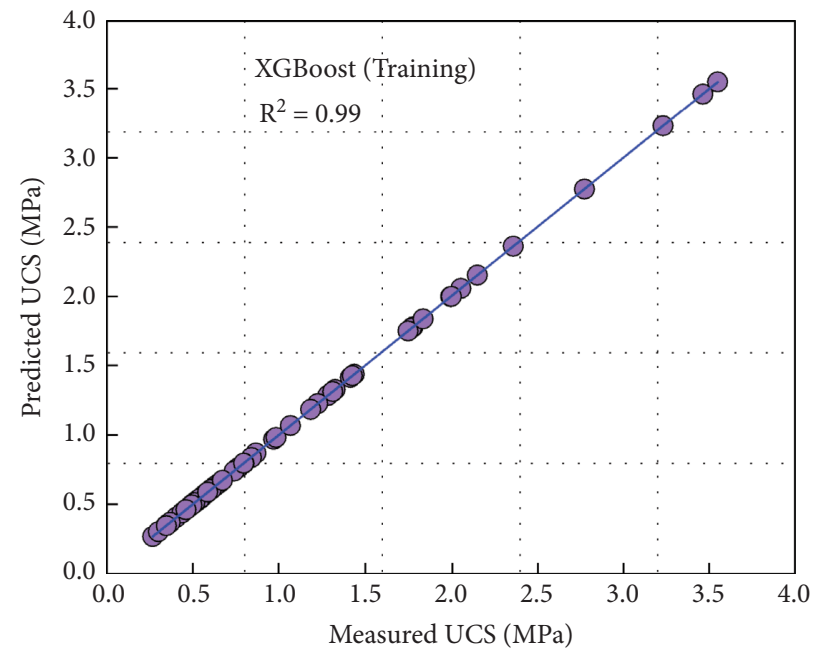

(d)

FIGURE 9: Scatter plots of predicted UCS as opposed to measured UCS of different models in the training stage: (a) GBR, (b) Catboost, (c) LightGBM, and (d) XGBoost. 


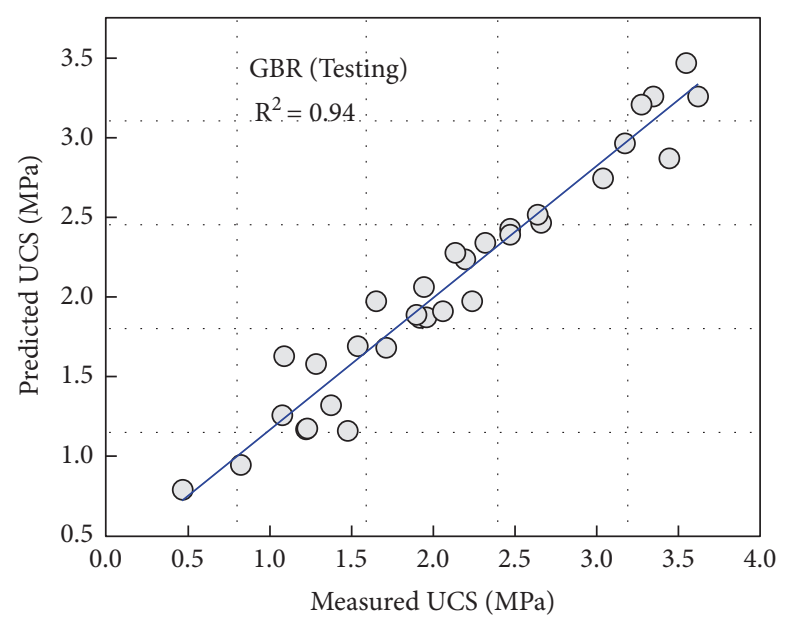

(a)

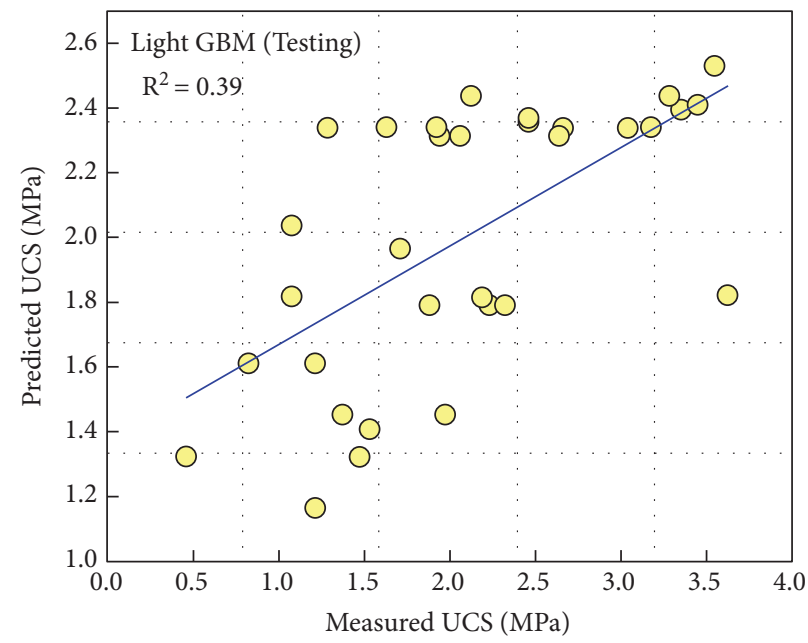

(c)

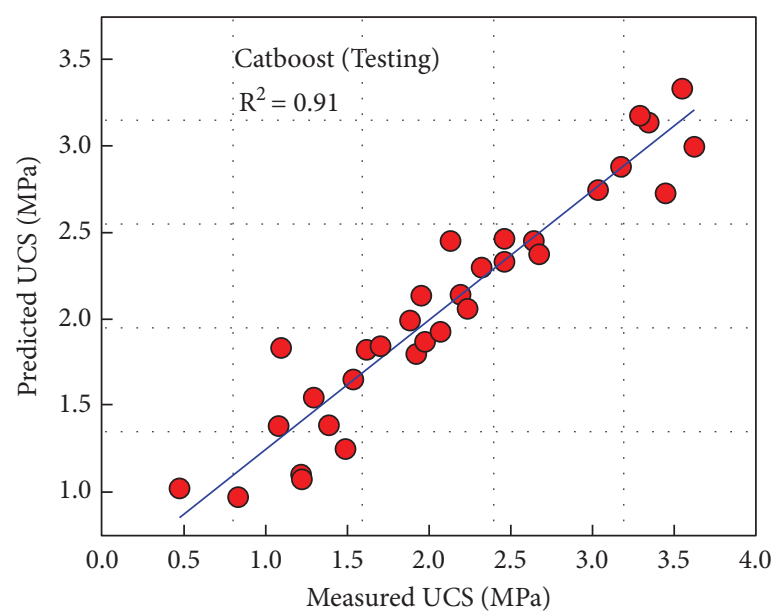

(b)

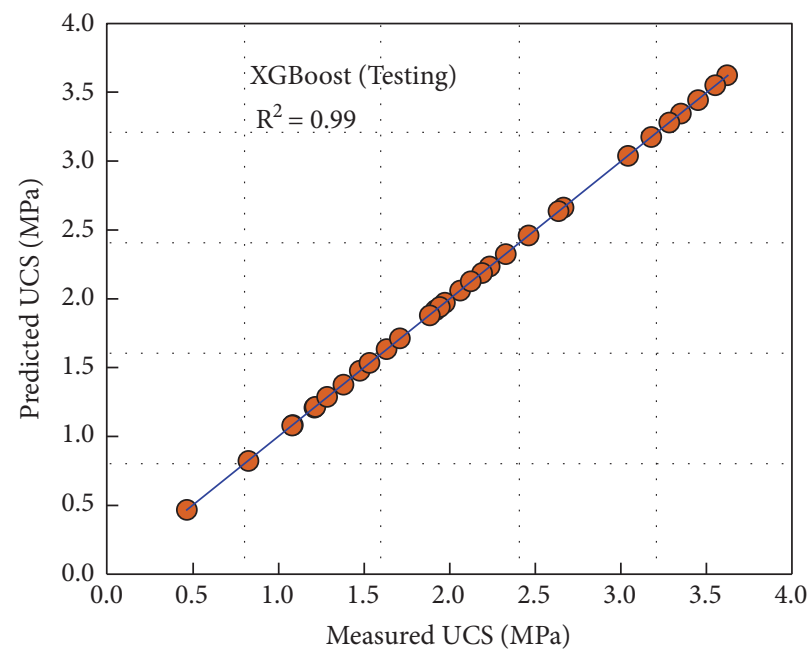

(d)

FigURE 10: Scatter plots of predicted UCS as opposed to measured UCS of different models in the testing stage: (a) GBR, (b) Catboost, (c) LightGBM, and (d) XGBoost.

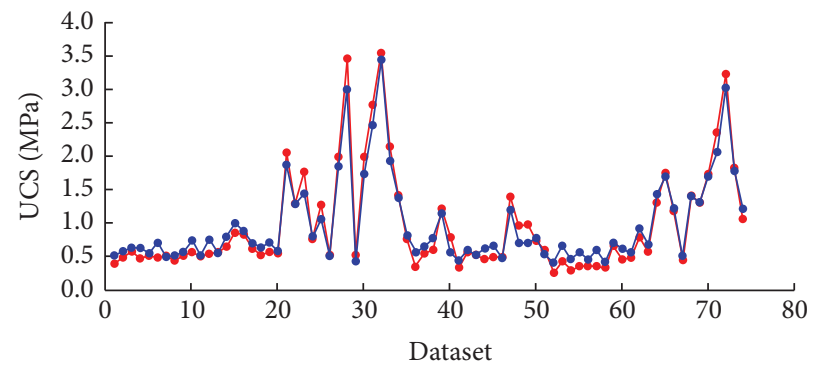

- Measured

$\rightarrow$ GBR

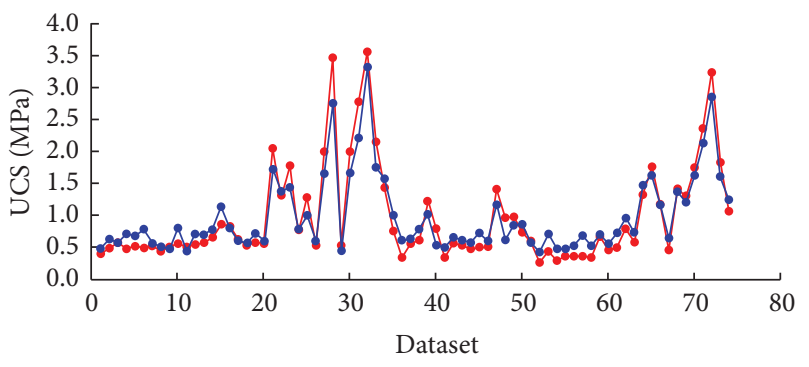

$\rightarrow$ Measured

$\rightarrow$ Catboost

(a)

(b)

Figure 11: Continued. 


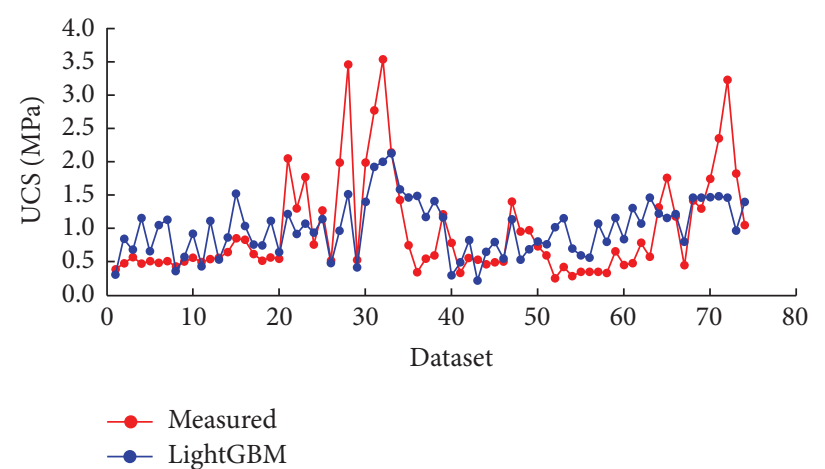

(c)

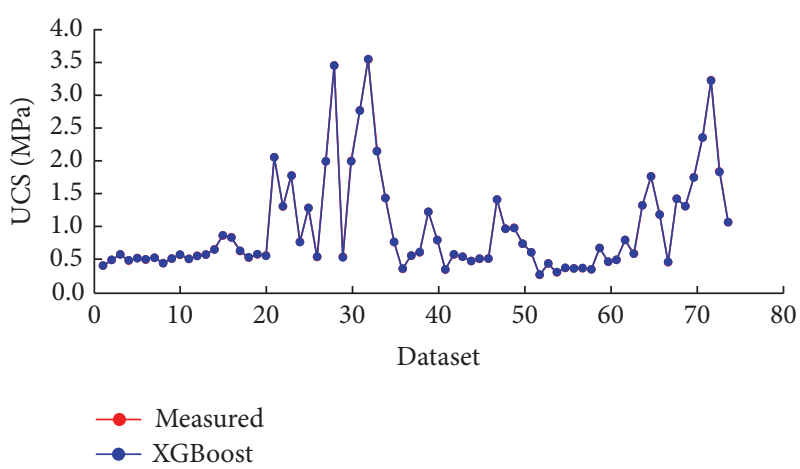

(d)

FIGURE 11: Performance plots of predicted UCS as opposed to measured UCS in the training stage phase: (a) GBR, (b) Catboost, (c) LightGBM, and (d) XGBoost.

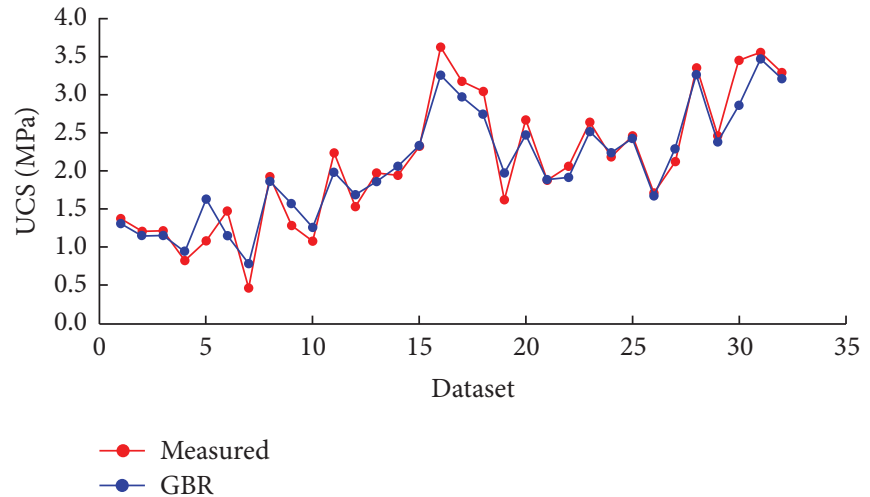

(a)

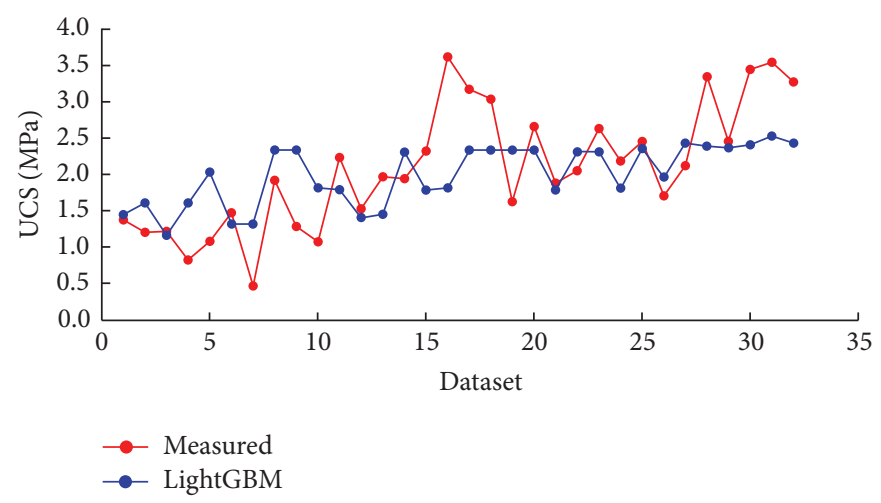

(c)

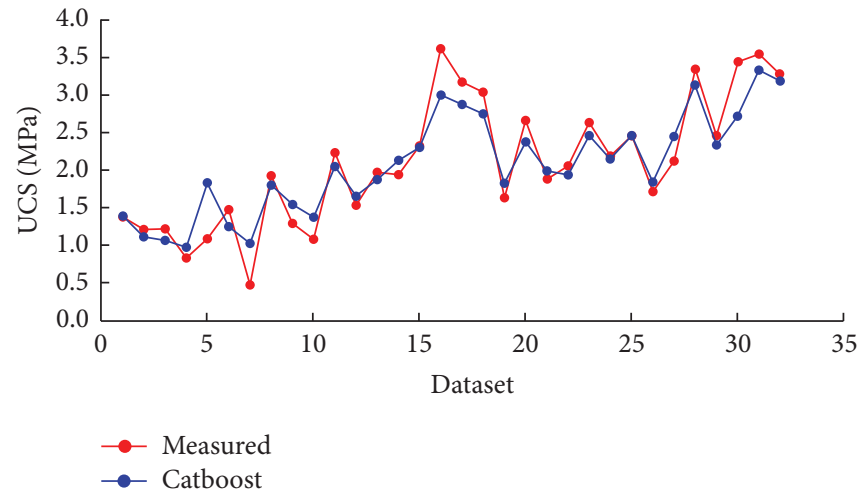

(b)

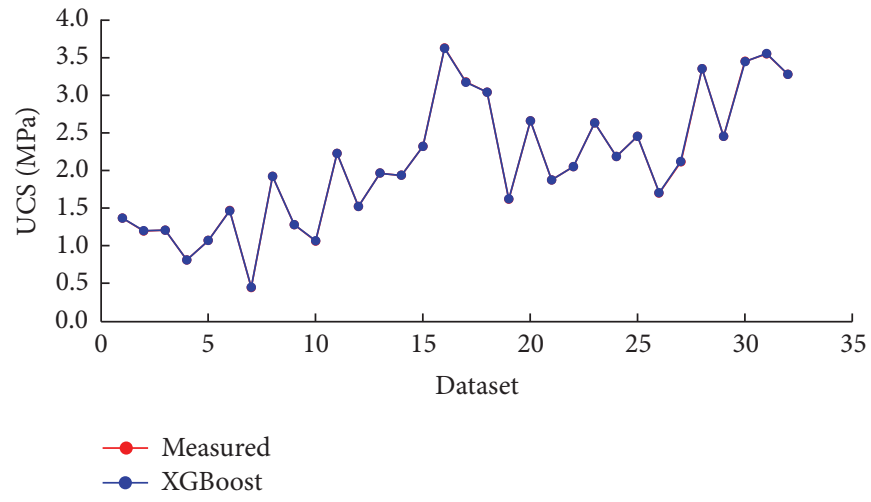

(d)

FIgURE 12: Performance plots of predicted UCS as opposed to measured UCS in the testing stage phase: (a) GBR, (b) Catboost, (c) LightGBM, and (d) XGBoost.

The results of the present study are similar to a previous study in the literature where Zhong et al. [40] also developed the XGBoost algorithm for predicting of rock mechanical properties, i.e., UCS, in their study, which proved to have the best performance in results with maximum $R^{2}$ and smallest MAE and RMSE. 

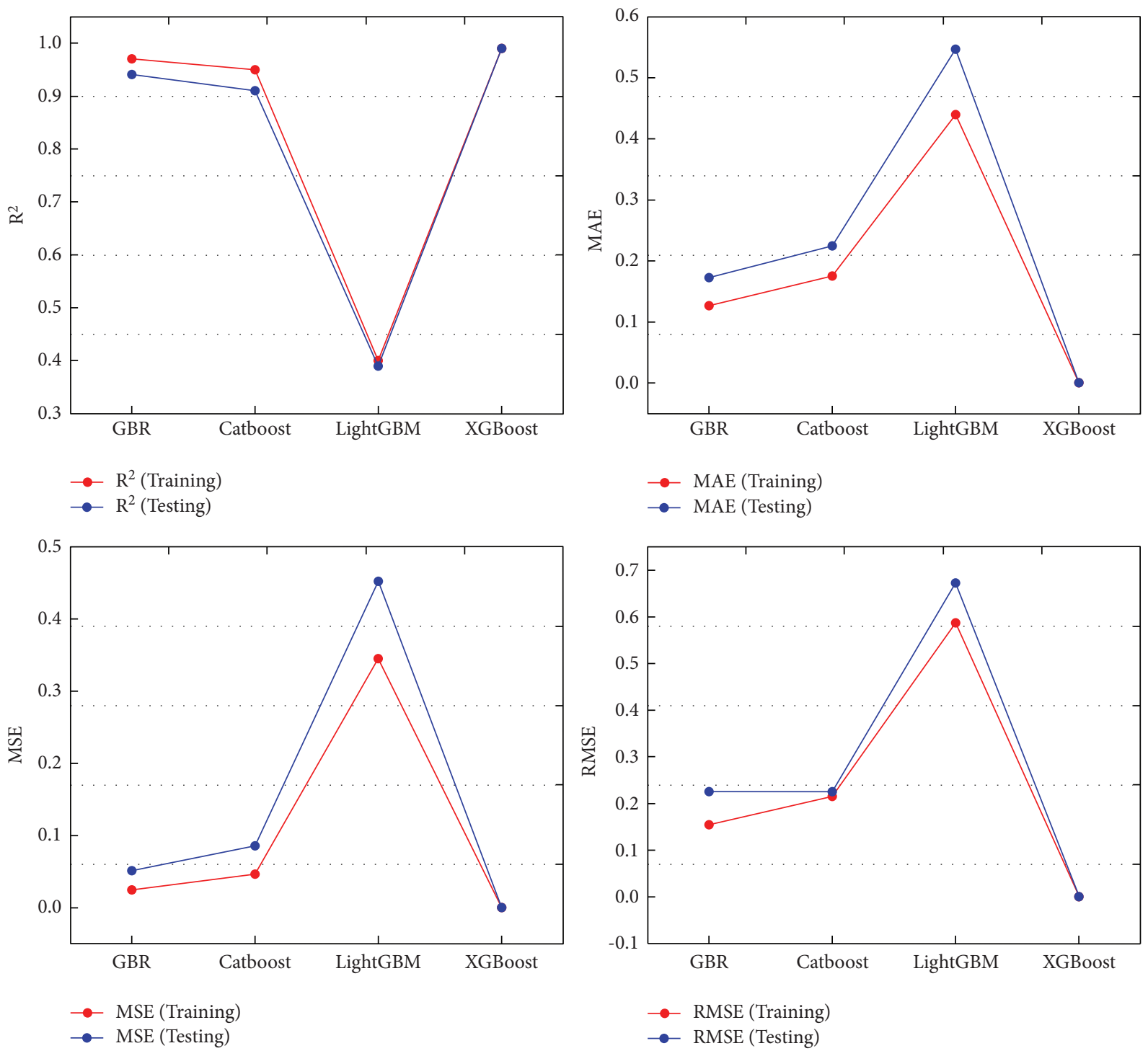

FIGURE 13: Evaluation criteria of the proposed algorithms $R^{2}$, MAE, MSE, and RMSE in the training phase and testing phase, respectively.

TABLE 9: Performance indices of the proposed algorithms in this study.

\begin{tabular}{|c|c|c|c|c|c|c|c|c|}
\hline \multirow{2}{*}{ Proposed algorithms } & \multicolumn{4}{|c|}{ Training } & \multicolumn{4}{|c|}{ Testing } \\
\hline & $R^{2}$ & MAE & MSE & RMSE & $R^{2}$ & MAE & MSE & RMSE \\
\hline GBR & 95.7146 & 0.1266 & 0.0241 & 0.1554 & 92.7812 & 0.1726 & 0.0507 & 0.2253 \\
\hline Catboost & 91.7856 & 0.17521 & 0.0463 & 0.2152 & 87.8611 & 0.2244 & 0.0853 & 0.2921 \\
\hline LightGBM & 38.8687 & 0.43944 & 0.3448 & 0.5872 & 35.7219 & 0.5468 & 0.4521 & 0.6723 \\
\hline XGBoost & 99.99 & 0.00062 & 0.00000062 & 0.00079 & 99.99 & 0.00054 & 0.0000047 & 0.00069 \\
\hline
\end{tabular}

\section{Sensitivity Analysis}

Sensitivity analysis is a statistical tool that evaluates how target features are influenced based on changes in input features [67]. In this work, sensitivity analysis has been carried out by implementing "feature importance" module in python programing language on the superior performed XGBoost testing data. The following equation [68] depicts the formula of the feature importance:

$$
\text { XGboostfm }_{m}=\frac{\sum_{\mathrm{l} \in \text { alltree }} \mathrm{fm}_{\mathrm{mn}}}{T},
$$

where XGboostfm $\mathrm{I}_{m}=$ the importance of feature $m$ estimated from all trees in the XGBoost testing dataset, $\mathrm{fm}_{\mathrm{mn}}=$ the feature importance for $m$ tree in $n$, and $T$ indicates the entire number of trees.

From Figure 15, it is clear that BTS and $\rho_{\mathrm{w}}$ are positively correlated, and the moisture and $\rho_{\mathrm{d}}$ are negatively correlated 


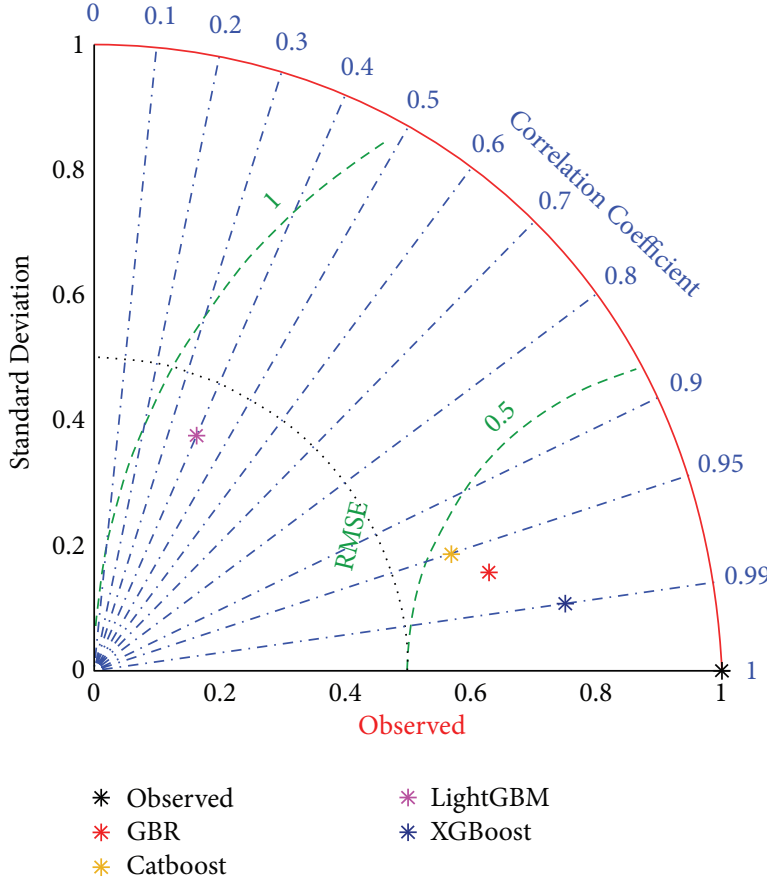

(a)

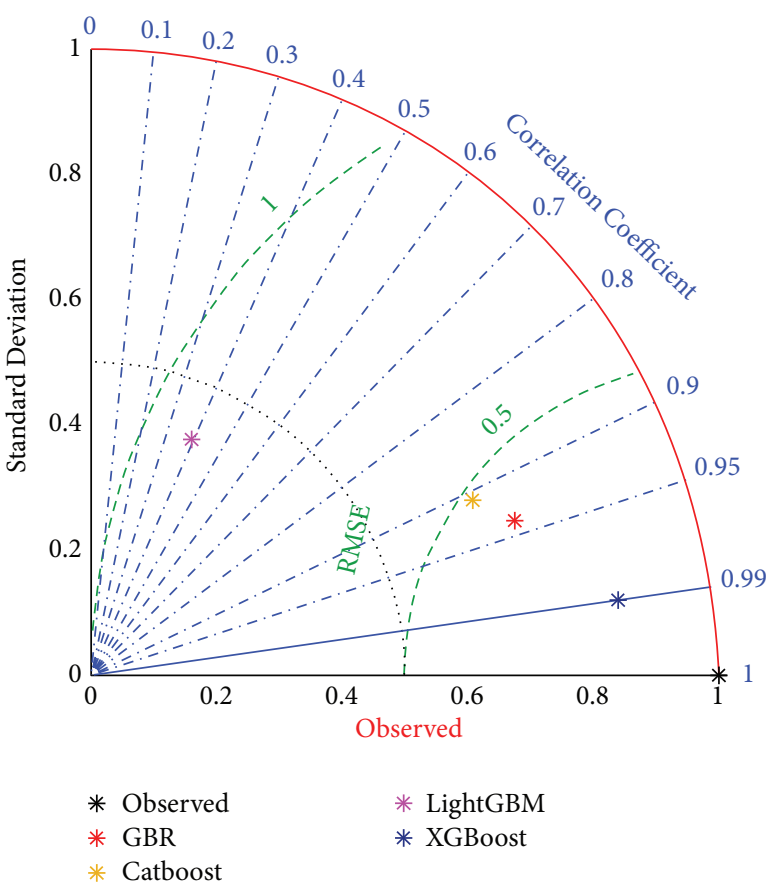

(b)

FIGURE 14: Taylor diagrammatic representation of GBR, XGBoost, Catboost, and LightGBM models in the (a) training phase and (b) testing phase.

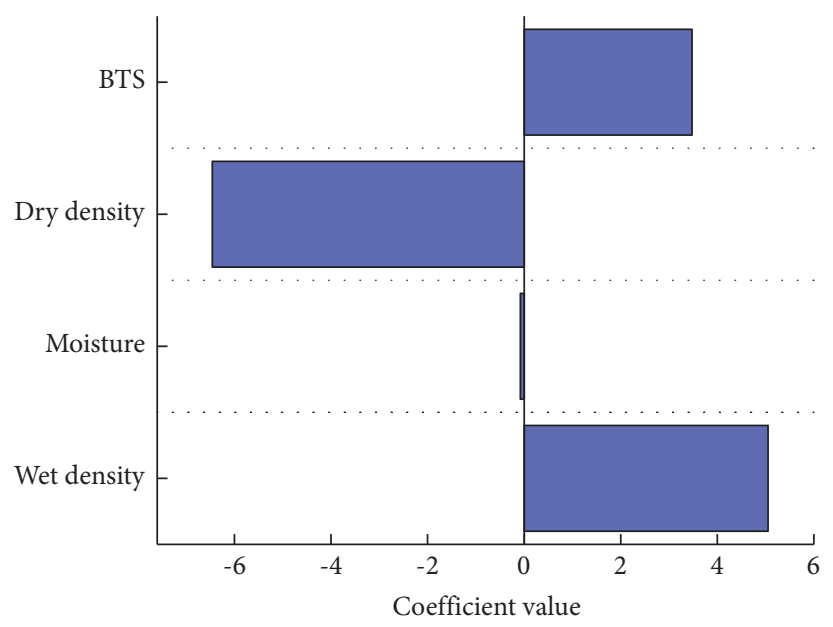

FIGURE 15: Sensitivity analysis of UCS dataset.

with the UCS. The feature importance of each input feature is given as:

$\mathrm{BTS}=5.050, \quad \rho_{\mathrm{w}}=-0.078, \quad$ moisture $=-6.457, \quad$ and $\rho_{\mathrm{d}}=3.475$.

\section{Conclusion}

UCS is one of the significant rock strength parameters and has various applications in mining, geology, geotechnical engineering, etc. An advanced data visualization model has been established in order to depict the research findings of various indirect tests to UCS. This study developed the four gradient boosting machine learning algorithms such as GBR, Catboost, LightGBM, and XGBoost for predicting UCS of soft sedimentary rocks of Block-IX at Thar Coalfield. The application of these developed algorithms has rarely been employed for the prediction of rock mechanical parameters, especially UCS. In order to execute the gradient boosting machine learning algorithms, the four significant input parameters $\rho \mathrm{w}$ in $\mathrm{g} / \mathrm{cm}^{3}$; moisture in $\%$; dry $\rho \mathrm{d}$ in $\mathrm{g} / \mathrm{cm}^{3}$; and BTS in MPa are used as input parameter and the corresponding UCS is employed as output parameter. Moreover, 106-point dataset was split identically for each algorithm into $70 \%$ for the training phase and $30 \%$ for the testing 
phase. The developed models were executed in python programing language. In this study, the performance of each developed algorithm was computed and as a result, XGBoost outperformed the GBR, Catboost, and LightGBM with $R^{2}=0.99, \quad \mathrm{MAE}=0.00062, \quad \mathrm{MSE}=0.0000006, \quad$ and $\mathrm{RMSE}=0.00079$ in the training phase and $R^{2}=0.99$, $\mathrm{MAE}=0.00054, \mathrm{MSE}=0.0000005$, and RMSE $=0.00069$ in the testing phase. In addition, according to the Taylor diagram, the standard deviation of the XGBoost is nearest to the original value of the standard deviation. Therefore, the XGBoost exhibits high accuracy in the training and testing phases, respectively. In this study, the developed XGBoost algorithm proved to be the best-fit algorithm for predicting the UCS of soft sedimentary rocks of the Block-IX at Thar Coalfield, Pakistan. Future work can be expanded employing different datasets to verify the more accuracy of the proposed algorithms.

\section{Data Availability}

The data, models, and code generated and/or employed during the study are available from the corresponding author upon request.

\section{Conflicts of Interest}

The authors declare no potential conflicts of interest.

\section{Acknowledgments}

This research was supported by the Science and Technology Innovation Project of Guizhou Province (Qiankehe Platform Talent [2019] 5620 to XZ).

\section{References}

[1] D. C. Washington, Limitations of Rock Mechanics in EnergyResource Recovery and Development, National Academy of Science, Washington, DC, USA, 1978.

[2] S. Demirdag, K. Tufekci, R. Kayacan, H. Yavuz, and R. Altindag, "Dynamic mechanical behavior of some carbonate rocks," International Journal of Rock Mechanics and Mining Sciences, vol. 47, no. 2, pp. 307-312, 2010.

[3] D. Jahed Armaghani, E. Tonnizam Mohamad, E. Momeni, M. S. Narayanasamy, and M. F. Mohd Amin, "An adaptive neuro-fuzzy inference system for predicting unconfined compressive strength and Young's modulus: a study on Main Range granite," Bulletin of Engineering Geology and the Environment, vol. 74, no. 4, pp. 1301-1319, 2015.

[4] E. Hakan and D. Kanik, "Multicriteria decision-making analysis based methodology for predicting carbonate rocks' uniaxial compressive strength," Earth Sciences Research Journal, vol. 16, no. 1, pp. 65-74, 2012.

[5] D. J. Armaghani, E. T. Mohamad, E. Momeni, M. Monjezi, and M. S. Narayanasamy, "Prediction of the strength and elasticity modulus of granite through an expert artificial neural network," Arabian Journal of Geosciences, vol. 9, no. 1, pp. 1-16, 2016.

[6] Á. Török and B. Vásárhelyi, "The influence of fabric and water content on selected rock mechanical parameters of travertine, examples from Hungary," Engineering Geology, vol. 115, no. 34, pp. 237-245, 2010.
[7] N. Yilmaz, "The influence of testing procedures on uniaxial compressive strength prediction of carbonate rocks from Equotip hardness tester (EHT) and proposal of a new testing methodology: hybrid dynamic hardness (HDH)," Rock Mechanics and Rock Engineering, vol. 46, no. 1, pp. 95-106, 2013.

[8] J. S. Lee, L. Smallwood, and E. Morgan, "New application of rebound hardness numbers to generate logging of unconfined compressive strength in laminated shale formations," in Proceedings of the 48th US rock mechanics/geomechanics symposium, (ARMA), vol. 2, pp. 972-978, Minneapolis, MN, USA, June 2014.

[9] F. Meulenkamp and M. A. Grima, "Application of neural networks for the prediction of the unconfined compressive strength (UCS) from Equotip hardness," International Journal of Rock Mechanics and Mining Sciences, vol. 36, no. 1, pp. 29-39, 1999.

[10] H. Aoki and Y. Matsukara, "Estimating the unconfined compressive strength of intact rocks from Equotip hardness," Bulletin of Engineering Geology and the Environment, vol. 67, no. 1, pp. 23-29, 2008.

[11] W. Verwaal and A. Mulder, "Estimating rock strength with the Equotip hardness tester: technical note," International Journal of Rock Mechanics and Mining Science \& Geomechanics Abstracts, vol. 30, no. 6, pp. 659-662, 1993.

[12] I. Cobanoglu and S. Celik, "Estimation of uniaxial compressive strength from point load strength, Schmidt hardness and P-wave velocity," Bulletin of Engineering Geology and the Environment, vol. 67, no. 4, pp. 491-498, 2008.

[13] A. K. Ghose and S. Chakraborti, "Empirical strength indices of Indian coals," in Proceedings of the 27th US symposium on rock mechanics Balkema, Rotterdam, Netherlands, June 1986.

[14] A. Azimian, R. Ajalloeian, and L. Fatehi, "An Empirical correlation of uniaxial compressive strength with $\mathrm{P}$-wave velocity and point load strength index on Marly rocks using statistical method," Geotechnical \& Geological Engineering, vol. 32, no. 1, pp. 205-214, 2014.

[15] O. Katz, Z. Reches, and J. C. Roegiers, "Evaluation of mechanical rock properties using a Schmidt Hammer," International Journal of Rock Mechanics and Mining Sciences, vol. 37, no. 4, pp. 723-728, 2000.

[16] M. M. Aliyu, J. Shang, W. Murphy et al., "Assessing the uniaxial compressive strength of extremely hard cryptocrystalline flint," International Journal of Rock Mechanics and Mining Sciences, vol. 113, pp. 310-321, 2019.

[17] B. Tiryaki, "Predicting intact rock strength for mechanical excavation using multivariate statistics, artificial neural networks and regression trees," Engineering Geology, vol. 99, no. 1-2, pp. 51-60, 2008.

[18] K. Diamantis, E. Gartzos, and G. Migiros, "Study on uniaxial compressive strength, point load strength index, dynamic and physical properties of serpentinites from central greece: test results and empirical relations," Engineering Geology, vol. 108, no. 3-4, pp. 199-207, 2009.

[19] T. Morales, G. Uribe-Etxebarria, J. A. Uriarte, and I. F. Valderrama, "Geomechanical characterisation of rock masses in Alpine regions: the Basque Arc (Basque-Cantabrian basin, Northern Spain)," Engineering Geology, vol. 71, no. 3-4, pp. 343-362, 2004.

[20] S. Kahraman and O. Gunaydin, "The effect of rock classes on the relation between uniaxial compressive strength and point load index," Bulletin of Engineering Geology and the Environment, vol. 68, no. 3, pp. 345-353, 2009.

[21] D. C. Entwisle, R. N. Hobbs, L. D. Jones, D. Gunn, and M. G. Raines, "The relationship between effective porosity, 
uniaxial compressive strength and sonic velocity of intact Borrowdale volcanic group core samples from Sella field," Geotechnical \& Geological Engineering, vol. 23, no. 6, pp. 793-809, 2005.

[22] E. S. Lindquist and R. E. Goodman, "Strength and deformation properties of a physical model mélange," in Proceedings of the 1st North American Rock Mechanics Symposium, P. P. Nelson and S. E. Laubach, Eds., Balkema, Rotterdam, 1994.

[23] Y. Abdi, A. T. Garavand, and R. Z. Sahamieh, "Prediction of strength parameters of sedimentary rocks using artificial neural networks and regression analysis," Arabian Journal of Geosciences, vol. 11, no. 19, pp. 1-11, 2018.

[24] A. Teymen and E. C. Mengüç, "Comparative evaluation of different statistical tools for the prediction of uniaxial compressive strength of rocks," International Journal of Mining Science and Technology, vol. 30, no. 6, pp. 785-797, 2020.

[25] A. F. Cabalar, A. Cevik, and C. Gokceoglu, "Some applications of adaptive neuro-fuzzy inference system (ANFIS) in geotechnical engineering," Computers and Geotechnics, vol. 40, pp. 14-33, 2012.

[26] J. Cao, J. Gao, H. N. Rad, A. S. Mohammed, M. Hasanipanah, and J. Zhou, "A novel systematic and evolved approach based on XGBoost-firefly algorithm to predict Young's modulus and unconfined compressive strength of rock," Engineering with Computers, pp. 1-17, 2021.

[27] F. Yang, Z. Li, Q. Wang et al., "Cluster-formula-embedded machine learning for design of multicomponent $\beta$-Ti alloys with low Young's modulus," Npj Computational Materials, vol. 6 , no. 1, pp. 1-11, 2020.

[28] J. Duan, P. G. Asteris, H. Nguyen, X. N. Bui, and H. Moayedi, "A novel artificial intelligence technique to predict compressive strength of recycled aggregate concrete using ICAXGBoost model," Engineering with Computers, vol. 37, pp. 1-18, 2020.

[29] D. Jahed Armaghani, E. Tonnizam Mohamad, M. Hajihassani, S. Yagiz, and H. Motaghedi, "Application of several nonlinear prediction tools for estimating uniaxial compressive strength of granitic rocks and comparison of their performances," Engineering with Computers, vol. 32, no. 2, pp. 189-206, 2016.

[30] A. Jamshidi, M. R. Nikudel, M. Khamehchiyan, and R. Z. Sahamieh, "The effect of specimen diameter size on uniaxial compressive strength, $\mathrm{P}$-wave velocity and the correlation between them," Geomechanics and Geoengineering, vol. 11, no. 1, pp. 13-19, 2016.

[31] S. Chehreh Chelgani, S. S. Matin, and J. C. Hower, "Explaining relationships between coke quality index and coal properties by Random Forest method," Fuel, vol. 182, pp. 754-760, 2016.

[32] Y. Abdi and A. Taheri-Garavand, "Application of the ANFIS approach for estimating the mechanical properties of sandstones," Emirates Journal for Engineering Research, vol. 25, no. 4 , p. $1,2020$.

[33] N. Ceryan and P. Samui, "Application of soft computing methods in predicting uniaxial compressive strength of the volcanic rocks with different weathering degree," Arabian Journal of Geosciences, vol. 13, no. 7, pp. 1-18, 2020.

[34] N. Ceryan and N. K. Can, "Prediction of the uniaxial compressive strength of rocks materials," in Handbook of Research on Trends and Digital Advances in Engineering Geology, pp. 31-96, Igi Globa, Hershey, Pennsylvania, 2018.

[35] M. Monjezi, H. Amini Khoshalan, and M. Razifard, "A neurogenetic network for predicting uniaxial compressive strength of rocks," Geotechnical \& Geological Engineering, vol. 30, no. 4, pp. 1053-1062, 2012.

[36] S. Aboutaleb, M. Behnia, R. Bagherpour, and B. Bluekian, "Using non-destructive tests for estimating uniaxial compressive strength and static Young's modulus of carbonate rocks via some modeling techniques," Bulletin of Engineering Geology and the Environment, vol. 77, no. 4, pp. 1717-1728, 2018.

[37] E. Ghasemi, H. Kalhori, R. Bagherpour, and S. Yagiz, "Model tree approach for predicting uniaxial compressive strength and Young's modulus of carbonate rocks," Bulletin of Engineering Geology and the Environment, vol. 77, no. 1, pp. 331-343, 2018.

[38] R. Barzegar, M. Sattarpour, R. Deo, E. Fijani, and J. Adamowski, "An ensemble tree-based machine learning model for predicting the uniaxial compressive strength of travertine rocks," Neural Computing \& Applications, vol. 32, no. 13, pp. 9065-9080, 2020.

[39] S. S. Matin, L. Farahzadi, S. Makaremi, S. C. Chelgani, and G. Sattari, "Variable selection and prediction of uniaxial compressive strength and modulus of elasticity by random forest," Applied Soft Computing, vol. 70, pp. 980-987, 2018.

[40] R. Zhong, M. Tsang, G. Makusha, B. Yang, and Z. Chen, "Improving rock mechanical properties estimation using machine learning," in Proceedings of the 2021 Resource Operators Conference, Mining Engineering, University of Wollongong, Wollongong, Australia, February 2021.

[41] H. T. T. Ngo, T. A. Pham, H. L. T. Vu, and L. V. Giap, "Application of artificial intelligence to determined unconfined compressive strength of cement-stabilized soil in vietnam," Applied Sciences, vol. 11, no. 4, p. 1949, 2021.

[42] S. B. H. Saeed and Y. M. Alshkane, "Effect of freezing and thawing on physical and mechanical properties of sedimentary rock," Journal of Garmian University, vol. 5, no. 2, pp. 155-172, 2018.

[43] E. T. Mohamad, D. Jahed Armaghani, E. Momeni, and S. V. Alavi Nezhad Khalil Abad, "Prediction of the unconfined compressive strength of soft rocks: a PSO-based ANN approach," Bulletin of Engineering Geology and the Environment, vol. 74, no. 3, pp. 745-757, 2015.

[44] N. M. Shahani, Z. Wan, L. Guichen et al., "Numerical analysis of top coal recovery ratio by using discrete element method," Pakistan Journal of Engineering and Applied Sciences, vol. 24, no. 1, pp. 26-35, 2019.

[45] N. M. Shahani, Z. Wan, X. Zheng et al., "Numerical modeling of longwall top coal caving method at thar coalfield," Journal of Metals, Materials and Minerals, vol. 30, no. 1, pp. 57-72, 2020.

[46] E. T. Brown, "Rock characterization testing \& monitoring ISRM suggested methods," ISRM - International Society for Rock Mechanics, p. 211, Pergamon Press, London, 2007.

[47] ASTM - American Society for Tenting and Materials, D454385:, Standard Practices for Preparing Rock Core as Cylindrical Test Specimens and Verifying Conformance to Dimensional and Shape Tolerances, ASTM International, West Conshohocken, Pennsylvania, 2013.

[48] Y. Freund, R. Schapire, and N. Abe, "A short introduction to boosting," Journal of Japanese Society for Artificial Intelligence, vol. 14, no. 5, pp. 771-780, 1999.

[49] R. E. Schapire, "The strength of weak learnability," Machine Learning, vol. 5, no. 2, pp. 197-227, 1990.

[50] M. Kearns, Thoughts on Hypothesis Boosting, Machine Learning Class Project, University of Pennsylvania, Philadelphia, PA, USA, 1988. 
[51] J. H. Friedman, "Greedy function approximation: a gradient boosting machine," Annals of Statistics, vol. 29, pp. 11891232, 2001.

[52] J. Friedman, T. Hastie, and T. Robert, "The elements of statistical learning," Springer Series in Statistics, vol. 1, no. 10, 2001.

[53] J. H. Friedman, "Stochastic gradient boosting," Computational Statistics \& Data Analysis, vol. 38, no. 4, pp. 367-378, 2002.

[54] A. V. Dorogush, V. Ershov, and A. Gulin, CatBoost: Gradient Boosting with Categorical Features Support, arXiv preprint arXiv:1810.11363, 2018.

[55] L. Prokhorenkova, G. Gusev, A. Vorobev, A. V. Dorogush, and A. Gulin, "CatBoost: unbiased boosting with categorical features," in Proceedings of the Advances in Neural Information Processing Systems, pp. 6638-6648, Curran Associates Inc., Montréal, QC, Canada, December 2018.

[56] G. Ke, Q. Meng, T. Finley et al., "Lightgbm: a highly efficient gradient boosting decision tree," Advances in Neural Information Processing Systems, vol. 30, pp. 3146-3154, 2017.

[57] H. Zeng, C. Yang, H. Zhang et al., "A lightGBM-based EEG analysis method for driver mental states classification," Computational Intelligence and Neuroscience, vol. 2019, Article ID 3761203, 2019.

[58] W. Liang, S. Luo, G. Zhao, and H. Wu, "Predicting hard rock pillar stability using GBDT, XGBoost, and LightGBM algorithms," Mathematics, vol. 8, no. 5, p. 765, 2020.

[59] Q. Meng, G. Ke, T. Wang et al., "A communication-efficient parallel algorithm for decision tree," in Proceedings of the 30th International Conference on Neural Information Processing Systems, pp. 1271-1279, Red Hook, NY, December 2016.

[60] S. Ranka and V. Singh, "Clouds: a decision tree classifier for large datasets," in Proceedings of the 4th Knowledge Discovery and Data Mining Conference, pp. 2-8, New York, NY, USA, August 1998.

[61] R. Jin and G. Agrawal, "Communication and memory efficient parallel decision tree construction," in Proceedings of the 2003 SIAM International Conference on Data Mining, pp. 119-129, SIAM, San Francisco, CA, USA, May 2003.

[62] G. Ke, Q. Meng, T. Finley et al., "Lightgbm: a highly efficient gradient boosting decision tree," in Advances in neural information processing systems, I Guyon, UV Luxburg, S Bengio et al., Eds., vol. 30, pp. 3146-3154, Curran Associates Inc., Long Beach, California, USA, 2017.

[63] M. Kamran, "A probabilistic approach for prediction of drilling rate index using ensemble learning technique," Journal of Mining and Environment, vol. 12, no. 2, pp. 327337, 2021.

[64] C. J. Willmott, "Some comments on the evaluation of model performance," Bulletin of the American Meteorological Society, vol. 63, no. 11, pp. 1309-1313, 1982.

[65] M. Kamran, "A state of the art catboost-based T-distributed stochastic neighbor embedding technique to predict backbreak at dewan cement limestone quarry," Journal of Mining and Environment, vol. 12, no. 3, pp. 679-691, 2021.

[66] K. E. Taylor, "Summarizing multiple aspects of model performance in a single diagram," Journal of Geophysical Research Atmosphere, vol. 106, no. D7, pp. 7183-7192, 2001.

[67] M. Kamran, S. Bacha, and N. Mohammad, "A stochastic model upgrading gold content in cyanide leaching using Monte Carlo simulation," Journal of Mining and Environment, vol. 12, no. 3, pp. 619-631.

[68] S. Ronaghan, "The mathematics of decision trees, random forest and feature importance in scikit-learn and spark," 2018, https://towardsdatascience.com/the-mathematics-of-decision -trees-random-forest-and-feature-importance-in-scikit-learn -and-spark-f2861df67e3. 